LA-UR-95-80

\title{
FUNCTIONAL INTEGRATION OVER GEOMETRIES
}

\author{
Emil Mottola \\ Theoretical Division T-8, \\ Los Alamos National Laboratory, \\ M. S. B285, Los Alamos, NM 87545, USA \\ Electronic Mail: EMIL@PION.LANL.GOV \\ (Contribution to the special issue of the \\ Journal of Mathematical Physics \\ on Functional Integration,
}

July, 1995)

\begin{abstract}
The geometric construction of the functional integral over coset spaces $\mathcal{M} / \mathcal{G}$ is reviewed. The inner product on the cotangent space of infinitesimal deformations of $\mathcal{M}$ defines an invariant distance and volume form, or functional integration measure on the full configuration space. Then, by a simple change of coordinates parameterizing the gauge fiber $\mathcal{G}$, the functional measure on the coset space $\mathcal{M} / \mathcal{G}$ is deduced. This change of integration variables leads to a Jacobian which is entirely equivalent to the Faddeev-Popov determinant of the more traditional gauge fixed approach in non-abelian gauge theory. If the general construction is applied to the case where $\mathcal{G}$ is the group of coordinate reparametrizations of spacetime, the continuum functional inte-
\end{abstract}


gral over geometries, i.e. metrics modulo coordinate reparameterizations may be defined. The invariant functional integration measure is used to derive the trace anomaly and effective action for the conformal part of the metric in two and four dimensional spacetime. In two dimensions this approach generates the Polyakov-Liouville action of closed bosonic non-critical string theory. In four dimensions the corresponding effective action leads to novel conclusions on the importance of quantum effects in gravity in the far infrared, and in particular, a dramatic modification of the classical Einstein theory at cosmological distance scales, signaled first by the quantum instability of classical de Sitter spacetime. Finite volume scaling relations for the functional integral of quantum gravity in two and four dimension $\mathrm{s}$ are derived, and comparison with the discretized dynamical triangulation approach to the integration over geometries are discussed. Outstanding unsolved problems in both the continuum definition and the simplicial approach to the functional integral over geometries are highlighted.

Typeset using REVTEX 


\section{INTRODUCTION}

\section{A. The Problem of Quantum Gravity}

The beginning of the twentieth century witnessed the birth of two great breakthroughs in theoretical physics, quantum mechanics and relativity. Each of these carries with it its own distinct conceptual and mathematical framework. By the middle of the century, special relativity had been incorporated successfully into quantum theory with the introduction of quantum field theory, though not until the technical difficulties associated with the handling of infinities and renormalization had been surmounted. The prototype of all renormalizable quantum field theories is quantum electrodynamics (QED), which is based on a $U(1)$ gauge invariance. The extension of the gauge principle to non-abelian groups were then to play a pivotal role in the development of successful renormalizable theories of the electroweak and strong interactions of particle physics. The standard model based on the gauge group $\mathcal{G}=S U(3) \times S U(2) \times U(1)$ is presently in agreement with all the known data. In parallel to these developments in particle physics, Wilson's renormalization group approach to critical phenomena in statistical systems transformed renormalization from an embarassing technical blemish of field theory to a positive virtue with genuine physical implications [1]. The important lesson of Wilson's approach is that renormalizable theories may play a privileged role in physics only because deviations from their predictions are naturally suppressed by inverse powers of a very large ultraviolet cutoff scale. The renormalizable gauge theories of the standard model, so successful for describing physics at intermediate to low energy scales may be only effective theories in the infrared. Gradually it has become clear that the functional integral approach offers a simple, yet powerful unifying approach to both quantization of gauge theories and the renormalization group ideas of Wilson.

So far left out of the successful (partial) unification of fundamental forces in the standard model and the parallel development of renormalization theory is gravitation. Correspond-

ingly, the geometric approach to physics so favored by Einstein was relegated to a secondary 
or even forgotten role in most of these developments. As we approach the end of the century it is clear that a consistent theory of quantum gravity in four dimensions must be regarded as the outstanding unrealized goal of theoretical physics.

The difficulties in constructing such a theory of quantum gravity are many, both at the conceptual and the technical level. Certainly the unrenormalizable ultraviolet divergences present in the Einstein theory are among the most serious of these difficulties. It is clear that this problem at the ultrashort Planck scale $\left(\sqrt{\frac{\hbar}{G c^{3}}} \simeq 10^{-33} \mathrm{~cm}\right.$.) requires the introduction of new physics, and cannot be solved by reformulating quantum general relativity in a different way. String theories, which attempt to solve this problem directly by greatly increasing the number of degrees of freedom at the ultraviolet scale, is certainly one possibility for this new physics. Unfortunately, despite prodigious efforts in the last decade, the technical obstacles in constructing a completely consistent string theory that agrees with known four dimensional physics (let alone makes unambiguous predictions for experimentally testing new physics) still seem very great. Yet, this effort has not been for naught, since at the very least it has brought to bear a wealth of new mathematical tools on the problem of quantizing gravity. One legacy of this development that is likely to survive in any consistent quantum theory with invariance under coordinate reparameterizations is the central role played by geometric methods, if only to extract cleanly the physical predictions of the theory from the artifacts of coordinate choices. That separation already presents something of an obstacle to the understanding in the classical Einstein theory. The importance of formulating the much more difficult problem of quantum gravity in a coordinate invariant language, thereby avoiding the cumbersome and distracting technical hurdles inherent in non-geometric approaches should not be underestimated. Since the path integral is based on the classical action, which is invariant under reparameterizations of coordinates, it is the natural approach to quantization of gravity in which general coordinate invariance can be maintained in its most transparent form.

In the past, path integral methods have suffered something of a bad reputation, primarily for the apparent difficulty in defining precisely integration over such a large function 
space as the space of all metrics. It seemed impossible to assign any definite meaning to such a notion without a discretization procedure which would inherently introduce some choice of coordinates and immediately wipe out any advantage of the functional integral approach to quantum gravity. Just such an invariant definition of functional integration over two-geometries, i.e. the coset space of two-metrics modulo diffeomorphisms $\mathcal{M} / \mathcal{G}$, and in particular a much clearer understanding of the functional measure on this space has been one important by-product of the geometric approach to string theories [2]. Later it was realized that essentially the same geometric construction could be taken over to the functional integral over four-geometries directly [4]. The main objection to the use of the path integral method in quantum gravity was thereby removed.

Parallel to these developments in the continuum definition of the path integral for systems with coordinate invariance there has also been progress in an alternative proposal to quantizing geometry, which has been called the dynamical triangulation approach [5]. Based on Regge's discretization of a continuum manifold by means of triangulation into a finite number of regular simplices, there are are no coordinates (or coordinate invariances) in this approach since one sums over distinct geometries from the very outset. Besides being a conceptually simple formulation well-suited to a systematic numerical study by computer, the main advantage of the dynamical triangulation approach is that one can bring all of the tools and intuition gleaned from finite dimensional statistical systems to bear on quantum gravity. Renormalization group methods could be applied to coordinate invariant systems in a meaningful way for the first time, and of course, there are no infinities or ill-defined functional measures in the discrete definition of the path integral. However, the infinite volume limit of this formulation must be investigated carefully, and equivalence with

the continuum functional integral has yet to be demonstrated. In two dimensions the situation seems reasonably well under control, because of the comparison to exact results in the continuum for (among other things) the finite area scaling behavior of the path integral over closed surfaces with fixed topology. The program of extending the dynamical triangulation approach to four dimensions has only just commenced in the last few years [6,7], 
and there the situation is far less well understood. In this regard, comparisons of numerical data with the recent predictions for the scaling behavior of the functional integral over fourgeometries with fixed topology should prove very illuminating [8]. The idea here is to make firm predictions at the infrared fixed point of the effective continuum theory, independently of the details at the ultrashort Planck scale, in the same way as Wilson's approach to critical phenomena are independent of the lattice cutoff. The functional integral over geometries is the natural tool for analyzing the finite volume scaling and infrared fixed point behavior of quantum gravity, making contact between the continuum and dynamical triangulation approaches most directly.

The main purpose of this article is to describe the covariant approach to the functional integral over geometries from a physicist's (quite nonrigorous) point of view. After introduction to the general idea of the geometric approach by means of the quantization of the relativistic point particle and non-abelian gauge theory, several applications of the covariant path integral are considered in some detail. While the finite temperature partition function for gauge theories and the conformal anomaly in gravitational backgrounds are not new results, it is hoped that their derivation by means of the covariant path integral will convince physicists of the simplicity and usefulness of the geometric approach to path integral quantization, while at the same time motivating a few of the more mathematically inclined readers to attempt to put these elegant but still heuristic methods on a firmer mathematical footing. For the benefit of the latter audience special attention will be paid to open issues and unsolved problems in the continuum definition of the functional integral. The extension of the same geometric method to the construction of the functional

integral over the coset space of metrics modulo coordinate diffeomorphisms is straightforward, and determines the continuum functional integration measure in the path integral approach to quantum gravity.

In four dimensions this approach leads to novel conclusions about quantum gravitational effects at large distances which are discussed next. By comparing the continuum predictions for the finite volume scaling behavior of the functional integral to the dynamical triangulation 
approach, I hope to communicate some sense of the excitement and anticipation at this frontier of quantum gravity, as the subject begins to emerge from the shadows of confusion and speculation into much sharper focus. The solution of the continuum functional measure problem, the development of numerical techniques in the dynamical triangulation approach, and the appearance of the infrared renormalization group in the form of finite volume scaling relations have introduced some badly needed mathematical clarity and physical intuition into a field with still very sparse prospects of experimental verification. Future progress will require an even closer and more fruitful collaboration between mathematical precision and physical intuition.

The invariant path integral formulation of the problem of quantum gravity as a sum over geometries seems to offer the best promise of a unifying bridge allowing just such a fertile interplay of apparently disparate ideas and techniques, not least because of the prospect of a third ally soon entering the field, in the form of numerical results.

\section{B. Basics of Path Integral Quantization}

To establish notation and orientation, let us begin by reviewing the path integral approach to non-relativistic quantum mechanics described by a Hamiltonian,

$$
H=\frac{p^{2}}{2 m}+V(x)
$$

where $p=\frac{\hbar}{i} \frac{\partial}{\partial x}$ in the position representation. The evolution of the wavefunction with arbitrary initial condition at time $t_{0}$ is described succintly by the time evolution operator $\mathcal{U}$

$$
\psi(x ; t)=\int_{-\infty}^{\infty} d x_{0} \mathcal{U}\left(x, x_{0} ; t-t_{0}\right) \psi\left(x_{0} ; t_{0}\right)
$$

where $\mathcal{U}$ satisfies the Schrödinger Eq.,

$$
H \mathcal{U}=i \frac{\partial}{\partial t} \mathcal{U}
$$

which has the solution, 


$$
\mathcal{U}\left(x, x_{0} ; t\right)=\left\langle x|\exp (-i H t)| x_{0}\right\rangle
$$

The path integral representation for this quantity is easily derived by first dividing the total time interval $t$ into $N$ equal partitions of length $\epsilon$ (with $t_{n}=n \epsilon$ and $\left.n=0,1, \ldots, N\right)$, and then inserting the identity operator in the form of an integration over complete sets of position eigenstates of the particle at each of the $N-1$ intermediate times. In this way we find that

$$
\mathcal{U}\left(x, x_{0} ; t\right)=\lim _{N \rightarrow \infty} \prod_{n=1}^{N}\left\{\int d x_{n}\left\langle x_{n}\left|\left(1-i \epsilon H\left(t_{n}\right)\right)\right| x_{n-1}\right\rangle\right\} \delta\left(x-x_{N}\right)
$$

Evaluating each of the $N$ matrix elements by again inserting the identity in the form of $N$ integrations over conjugate momentum eigenstates $\left|p_{n}\right\rangle$, and using $\langle p \mid x\rangle=(2 \pi)^{-1} \exp (-i p x)$ yields the desired phase space path integral representation,

$$
\mathcal{U}\left(x, x_{0} ; t\right)=\lim _{N \rightarrow \infty} \prod_{n=1}^{N}\left\{\int \frac{d x_{n} d p_{n}}{2 \pi} \exp \left\{-i \epsilon\left[\frac{p_{n}^{2}}{2 m}+V\left(x_{n}\right)\right]+i p_{n}\left(x_{n}-x_{n-1}\right)\right\}\right\} \delta\left(x-x_{N}\right) .
$$

An important point about this representation that should be stressed is that the canonical phase space measure appears with no arbitrariness whatsover, and the limit $N \rightarrow \infty$ exists for piecewise smooth potentials, so Eq. (1.6) is well-defined.

Further, since all of the $p_{n}$ integrations are Gaussian, they may be performed explicitly to arrive at the position space representation of the Feynman path integral,

$$
\mathcal{U}\left(x, x_{0} ; t\right)=\lim _{N \rightarrow \infty} \prod_{n=1}^{N}\left\{\int \frac{m d x_{n}}{2 \pi i \epsilon} \exp \left\{\frac{i m\left(x_{n}-x_{n-1}\right)^{2}}{2 \epsilon}-i \epsilon V\left(x_{n}\right)\right\}\right\} \delta\left(x-x_{N}\right) .
$$

Since the quantity in the exponent is precisely the time-discretized form of the classical action functional evaluated on all possible paths between the initial position $x_{0}$ and the final position $x$ in time $t$, Eq. (1.7) gives a completely well-defined, unambiguous meaning to the shorthand notation,

$$
\mathcal{U}\left(x, x_{0} ; t\right)=\int_{x(0)=x_{0}}^{x(t)=x}[\mathcal{D} x] \exp \{i S[x]\}, \quad S[x]=\int_{0}^{t} d u\left[\frac{m \dot{x}^{2}}{2}-V(x)\right] .
$$


If $t$ were replaced by $-i \tau$ this is exactly the Wiener path integral which is well studied in the mathematical and statistical mechanics literature [9].

Although the configuration space path integral is well-defined by the discretization of the time interval above, such a procedure is awkward to implement, and is not well-suited to relativistically covariant Lagrangians where time should play no preferred role. The situation is reminiscient of the Riemann definition of ordinary integration: it is important that such a definition exists but it is not how integration is most easily performed in practice. Instead of the Riemannian partition of the interval of integration and an awkward limiting process, it is much more convenient to invoke the fundamental theorem of calculus and integrate functions by finding their anti-derivative, completely bypassing any discretization or limiting procedure. It is at this point that one already encounters difficulties in path integration, since no Lebesque theory of functional integration is known, and even the conditions under which a "fundamental theorem of functional calculus" should hold are uncertain. In the present state of development of functional integration we are reduced to either numerical methods which rely on a discretization definition similar to (1.7) or to consideration of a few special cases. Indeed any "Table of Functional Integrals" would be very short, since only Gaussian functional integrals may be evaluated in closed form. However, for this special case it is possible to give an alternate definition of the continuum integral which generalizes nicely to covariant field theories, and which we now discuss.

For a harmonic oscillator action functional we may expand about the classical solution to quadratic order,

$$
S_{\text {quad }}=\frac{m}{2} \int_{0}^{t} d u\left[\left(\frac{d x}{d u}\right)^{2}-\omega^{2} x^{2}\right]=S_{c l}\left(x, x_{0} ; t\right)+\frac{m}{2} \int_{0}^{t} d u \xi \Delta_{\omega} \xi,
$$

where $\xi(u)=x(u)-x_{c l}(u)$ and $x_{c l}(u)$ satisfies the boundary conditions, $x_{c l}(0)=x_{0}$ and $x_{c l}(t)=x$. The differential operator $\Delta_{\omega}$ is given by

$$
\Delta_{\omega}=-\frac{d^{2}}{d u^{2}}-\omega^{2}
$$

Since this operator may be diagonalized by finding a complete set of eigenfunctions $\xi_{n}(u)$ on the interval $[0, t]$ satisfying vanishing boundary conditions at the endpoints, we would 
like to be able to define the path integral over $\xi$ in terms of the determinant of $\Delta_{\omega}$ which is just the product of its eigenvalues, $\lambda_{n}$. This product is infinite, and requires a regulator, but just such a regulator is provided by the $\zeta$-function technique. Define

$$
\zeta\left(s \mid \Delta_{\omega}\right) \equiv \sum_{n}\left(\frac{\mu^{2}}{\lambda_{n}}\right)^{s}
$$

where $\mu$ is an arbitrary scale parameter. Eq. (1.11) defines a meromorphic function of $s$ with simple poles on the negative integers. In particular, the sum converges for $\operatorname{Re}(s)>1$, and this defines a function which may analytically continued to $s=0$. Since for a finite dimensional matrix operator,

$$
-\frac{d \zeta}{d s}\left(s=0 \mid \Delta_{\omega}\right)=\sum_{n} \ln \left(\frac{\lambda_{n}}{\mu^{2}}\right)=\ln \operatorname{det}\left(\frac{\Delta_{\omega}}{\mu^{2}}\right),
$$

we may use Eq. (11.12) to define the determinant of a differential operator with an infinite spectrum. Then the Gaussian path integral (1.7) with action (1.9) is defined (up to the arbitrary parameter $\mu$ ) by

$$
\int_{x(0)=x_{0}}^{x(t)=x}[\mathcal{D} x] \exp \left\{i S_{\text {quad }}[x]\right\}=\operatorname{det}^{-\frac{1}{2}}\left(\frac{\Delta_{\omega}}{\mu^{2}}\right) \exp \left\{i S_{c l}\left(x, x_{0} ; t\right)\right\} .
$$

The $\zeta$-function is closely related to the heat kernel $K$ defined by

$$
K\left(\tau \mid \Delta_{\omega}\right) \equiv \sum_{n} e^{-\lambda_{n} \tau}
$$

through

$$
\zeta\left(s \mid \Delta_{\omega}\right)=\frac{\mu^{2 s}}{\Gamma(s)} \int_{0}^{\infty} d \tau \tau^{s-1} K(\tau) .
$$

In general, it is easier to work with the $\zeta$-function when the differential operator has known eigenvalues, but with the heat kernel when deriving general properties of the determinant. Notice also that the definition of the functional determinant both agrees with the definition for finite dimensional matrices and has inherent in it a definition of a quadratic inner product on the space of functions, $\xi(u)$.

To illustrate the practicality of this definition let us evaluate the $\zeta$-function for the harmonic oscillator. The complete set of eigenfunctions of $\Delta_{\omega}$ satisfying the vanishing boundary conditions at the endpoints of the interval $[0, t]$ are easily found, 


$$
\xi_{n}(u)=\sin \left(\frac{\pi n u}{t}\right) \quad n=1,2, \ldots
$$

with the corresponding eigenvalues of $\Delta_{\omega}$ given by

$$
\lambda_{n}=\left(\frac{\pi n}{t}\right)^{2}-\omega^{2}
$$

Hence the $\zeta$-function (1.11) may be written as

$$
\begin{aligned}
\zeta\left(s \mid \Delta_{\omega}\right) & =\sum_{n=1}^{\infty}\left[\frac{\mu^{2}}{\left(\frac{\pi n}{t}\right)^{2}-\omega^{2}}\right]^{s} \\
& =\left(\frac{\mu t}{\pi}\right)^{2 s} \sum_{n=1}^{\infty} n^{-2 s}\left[1-\left(\frac{\omega t}{\pi n}\right)^{2}\right]^{-s}
\end{aligned}
$$

provided $\omega t$ is not $\pi n$ for any positive integer $n$. Let us assume that $\omega t<\pi$ for the sake of simplicity, although generalizations are straightforward. Using the binomial expansion for the last term in this expression, viz.,

$$
\left(1-x^{2}\right)^{-s}=\sum_{k=0}^{\infty} \frac{\Gamma(k+s)}{\Gamma(k+1) \Gamma(s)} x^{2 k}
$$

and interchanging the order of the $k$ and $n$ sums, we find that the sum over $n$ may be performed explicitly in terms of the Riemann zeta function $\zeta_{R}$ :

$$
\zeta\left(s \mid \Delta_{\omega}\right)=\left(\frac{\mu t}{\pi}\right)^{2 s} \sum_{k=0}^{\infty} \frac{\Gamma(k+s)}{\Gamma(k+1) \Gamma(s)}\left(\frac{\omega t}{\pi n}\right)^{2 k} \zeta_{R}(2 s+2 k) .
$$

This form is now suitable for analytic continuation towards $s=0$. Evaluating the first derivative there gives

$$
\left.\frac{d \zeta\left(s \mid \Delta_{\omega}\right)}{d s}\right|_{s=0}=2 \ln \left(\frac{\mu t}{\pi}\right) \zeta_{R}(0)+2 \zeta_{R}^{\prime}(0)+\sum_{k=1}^{\infty} \frac{1}{k}\left(\frac{\omega t}{\pi n}\right)^{2 k} \zeta_{R}(2 k) .
$$

The properties of $\zeta_{R}$ are well known. In addition to the values of the function and its derivative at $s=0$, the relationship of $\zeta_{R}(2 k)$ to the Bernoulli numbers is needed. The remaining sum is easily evaluated with the simple result:

$$
\left.\frac{d \zeta\left(s \mid \Delta_{\omega}\right)}{d s}\right|_{s=0}=\ln \left(\frac{\omega}{2 \mu \sin \omega t}\right) .
$$

Hence from the definition (1.12) 


$$
\operatorname{det}^{-\frac{1}{2}}\left(\frac{\Delta_{\omega}}{\mu^{2}}\right)=\exp \left(\left.\frac{1}{2} \frac{d \zeta\left(s \mid \Delta_{\omega}\right)}{d s}\right|_{s=0}\right)=\left(\frac{\omega}{2 \mu \sin \omega t}\right)^{\frac{1}{2}}
$$

and the path integral (1.13) has been evaluated unambiguously up to an overall normalization constant, without any partitioning of the time interval $[0, t]$ or the limiting procedure of the previous definition (1.7).

The unknown constant $\mu$ is somewhat analogous to a constant of integration. It can be determined from the normalization condition on $\mathcal{U}$ which follows from Eq. (1.4), namely

$$
\mathcal{U}\left(x, x_{0} ; t=0\right)=\delta\left(x-x_{0}\right)
$$

Equivalently one can compare the form of the time evolution operator obtained from Eqs. (1.7), (1.13) and (1.23) for the harmonic oscillator with the known result for the free particle with $\omega=0$. By either method one finds that $\mu$ must be replaced by $\frac{i \pi \hbar}{m}$ in order to give the correct answer. This is the same result for $\mathcal{U}$ that is obtained by the more laborious method of evaluating the discretized path integral expression Eq. (1.7) directly.

As a further exercise with the harmonic oscillator path integral, one can verify that substituting $t=-i \beta$ and $x_{0}=x$, and performing the Gaussian integration over $x$ gives the finite temperature partition function of the oscillator,

$$
Z(\beta)=\int_{-\infty}^{\infty} d x \mathcal{U}(x, x ;-i \beta)=\operatorname{Tr} e^{-\beta H}=\frac{1}{2 \sinh \left(\frac{\omega \beta}{2}\right)}
$$

This can be evaluated also by the $\zeta$-function method directly if one computes the determinant of $\Delta_{\omega}$ in Eq. (1.10) with periodic boundary conditions in imaginary time, $t=-i \beta$, otherwise following the same steps as Eqs. (1.16)-(1.23) above. In this case the parameter $\mu$ drops out of the final answer and the familiar result (1.25) is obtained directly.

The important lesson of these elementary examples is that a well-defined and practical definition of the continuum path integral exists, which does not require any discretization procedure, at least for Gaussian integrands. In this case one may pass from the Hamiltonian phase space path integral to the configuration space path integral defined entirely in the continuum by the proper definition of the functional determinant (1.12). This definition is 
the one which extends easily to systems with gauge or reparameterization invariance which is the main subject of this article.

\section{THE COVARIANT FUNCTIONAL INTEGRAL FOR SIMPLE SYSTEMS}

\section{A. Relativistic Point Particle}

There are several forms of the action for a relativistic point particle, all of which lead to the same answer. We shall use the form in flat Minkowski space,

$$
S[x ; N]=\frac{1}{2} \int_{0}^{1} d u\left(\frac{\dot{x}^{a} \eta_{a b} \dot{x}^{b}}{N}-m^{2} N\right) .
$$

This action possesses the reparameterization gauge invariance, $\mathcal{G}$

$$
\begin{aligned}
x^{a}(u) & \rightarrow x^{a}(f(u)), \\
N(u) & \rightarrow \frac{d f}{d u} N(f(u)),
\end{aligned}
$$

for any differentiable function $f(u)$ that leaves the endpoints of the interval $[0,1]$ fixed, i.e. provided

$$
f(0)=0, \quad f(1)=1 .
$$

The appearance of one factor of the determinant of the reparameterization, $u \rightarrow f(u)$ in the transformation of $N$ in Eq. (2.2) characterizes it as a density of weight one. The coordinate $x^{a}$ is a density of weight zero, i.e. a scalar under coordinate reparameterizations.

The momenta conjugate to $x^{a}$ and $N$ are:

$$
\begin{aligned}
p_{a} & \equiv \frac{\delta S}{\delta \dot{x}^{a}}=\frac{1}{N} \eta_{a b} \dot{x}^{b}, \quad \text { and } \\
p_{N} & \equiv \frac{\delta S}{\delta \dot{N}}=0
\end{aligned}
$$

respectively. Since the momentum conjugate to $N$ vanishes identically, the Hamiltonian system is a degenerate one with a primary first class constraint, in Dirac's nomenclature. The secondary first class constraint following from the primary one $p_{N}=0$ is 


$$
\dot{p}_{N}=-\frac{\delta S}{\delta N}=p_{a} \eta^{a b} p_{b}+m^{2} \equiv \mathcal{H}=0
$$

Because of this degeneracy, the phase space Hamiltonian path integral quantization cannot be applied without some modification. The correct modification is that of Batalin, Fradkin and Vilkovisky (BFV) [10]. Since the Hamiltonian $\mathcal{H}$ is quadratic in momenta, there is a simple coordinate space path integral obtained by integrating out the momenta in the BFV formalism. Like the non-relativistic example considered in the last section, the canonical path integral has a completely unambiguous well-defined measure in phase space, which defines a configuration space measure after the Gaussian integration over momenta. However, a careful definition of the measure obtained by integrating over the momentum variables seems to require again partitioning of the interval $[0,1]$ and a limiting procedure. An interesting open question is whether the passage from the BFV integral in phase space to the reparameterization invariant configuration space integral below is possible without recourse to discretization methods. Rather than entering into that discussion of the BFV phase space integral let us follow a different route and construct the configuration space path integral by geometric reparameterization invariance considerations alone «1, 11, 12].

The space of all configurations, $\mathcal{M}$ labelled by the functions $\left(x^{a}(u), N(u)\right)$ may be treated by methods borrowed from Riemannian geometry. At every "point" in the function space, $X^{i}$ we introduce the cotangent space, $\delta \mathcal{M}$ labelled by the basis vectors $\delta X^{i}=\left(\delta x^{a}(u), \delta N(u)\right)$. In Riemannian geometry one can introduce a metric on a space by defining a quadratic form, the line element, that maps $\delta \mathcal{M} \times \delta \mathcal{M}$ to the real numbers,

$$
(\delta s)^{2}=G_{i j}(X) \delta X^{i} \delta X^{j}
$$

The (infinitesimal) invariant volume measure of integration on the cotangent space, $\delta \mathcal{M}$

$$
d(\delta \mathcal{V})=\sqrt{\operatorname{det} G_{i j}(X)} d\left(\delta X^{1}\right) \times d\left(\delta X^{2}\right) \times \ldots
$$

may be chosen to satisfy the Gaussian normalization condition,

$$
\int d(\delta \mathcal{V}) \exp \left\{-\frac{1}{2} \delta X^{i} G_{i j}(X) \delta X^{j}\right\}=1
$$


and immediately induces an invariant volume measure on the full space $\mathcal{M}$,

$$
d \mathcal{V}=\sqrt{\operatorname{det} G_{i j}(X)} d X^{1} \times d X^{2} \times \ldots
$$

To define the path integral for a system possessing a reparameterization gauge invariance, we have only to define a quadratic inner product on the cotangent space $\delta \mathcal{M}$. Then the construction of the invariant measure on the function space of all configurations proceeds exactly along the lines of (2.7)-(2.10). Since the path integral is specified by an invariant action functional and an invariant integration measure, this procedure preserves the classical reparameterization invariance under quantization. Then by identifying configurations which differ only by a reparameterization of coordinates, we may integrate only over equivalence classes of coordinates, $\mathcal{M} / \mathcal{G}$ in a manifestly gauge or coordinate invariant way.

Let us illustrate this procedure for the relativistic point particle. Since both $N$ and $\delta N$ have weight one under (2.2), and

$$
\int_{0}^{1} N(u) d u \equiv \tau
$$

is invariant under reparameterizations (acting from the right), we can define an invariant inner product on $\mathcal{M}$ by

$$
\begin{aligned}
\langle\delta N, \delta N\rangle_{N} & \equiv \int_{0}^{1}\left(\frac{\delta N}{N}\right)^{2} N d u=\int_{0}^{1} \frac{(\delta N)^{2}}{N} d u \quad \text { and } \\
\langle\delta x, \delta x\rangle_{N} & \equiv \int_{0}^{1} \delta x^{a} G_{a b} \delta x^{b} d u=\int_{0}^{1} \delta x^{a} \eta_{a b} \delta x^{b} N d u
\end{aligned}
$$

since $\eta_{a b}$ is the only Lorentz invariant tensor possible in flat Minkowski space, up to a multiplicative constant, which may be absorbed into normalization of the measure by

$$
\begin{gathered}
\int[\mathcal{D}(\delta N)] \exp \left\{-\frac{1}{2}\langle\delta N, \delta N\rangle_{N}\right\}=1 \\
\int[\mathcal{D}(\delta x)] \exp \left\{-\frac{i}{2}\langle\delta x, \delta x\rangle_{N}\right\}=1
\end{gathered}
$$

A factor of $i$ is inserted into the second Gaussian because the Minkowski metric is pseudoRiemannian with one negative eigenvalue in the timelike direction, so that the volume form 
in (2.10) remains real. In Euclidean signature metrics this factor would be absent. These definitions generate an invariant functional measure on the full space $\mathcal{M}$ by eqs (2.7)-(2.10). In order to pull this measure back to an invariant measure on the quotient space of equivalence classes $\mathcal{M} / \mathcal{G}$, let us parameterize the gauge orbits $\mathcal{G}$ by the set of differentiable functions $f(u)$ satisfying the endpoint conditions, (2.3). Then an arbitrary lapse function $N(u)$ may be written in the form,

$$
N(u)=\tau \frac{d f}{d u} ; \quad f(u)=\frac{1}{\tau} \int_{0}^{u} N(u) d u
$$

In other words, the gauge equivalence class $\mathcal{M} / \mathcal{G}$ of all the functions $N(u)$ is characterized completely by the single parameter $\tau$ defined in Eq. (2.11), and the gauge fiber $\mathcal{G}$ is coordinatized by $f(u)$. Hence the integration measure on the quotient space is given by

$$
\frac{[\mathcal{D} N]}{[\mathcal{D} f]}[\mathcal{D} x]=J(\tau) d \tau[\mathcal{D} x]
$$

where $J$ is the Jacobian of change of variables from $N$ to $(\tau, f)$. The invariant path integral is then

$$
G\left(x, x_{0}\right)=\int J(\tau) d \tau \int_{x(0)=x_{0}}^{x(1)=x}[\mathcal{D} x] e^{i S[x ; \tau]}
$$

Our task now is to determine the Jacobian $J$. To do so write the cotangent space form of Eq. (2.16),

$$
\delta N=(\delta \tau) \frac{d f}{d u}+\tau \frac{d(\delta f)}{d u}
$$

and substitute this into the inner product definition (2.13). The cross term between $\delta \tau$ and $\delta f$ vanishes by using the endpoint conditions (2.3). Changing variables in the last term from $u$ to $v \equiv f(u)$ and defining

$$
\left.\xi(v) \equiv(\delta f)(u)\right|_{u=f^{-1}(v)}
$$

we obtain

$$
\langle\delta N, \delta N\rangle_{N}=\frac{(\delta \tau)^{2}}{\tau}+\tau \int_{0}^{1} d v \xi(v)\left[-\frac{d^{2}}{d v^{2}}\right] \xi(v)
$$


after an integration by parts. Now, it is straightforward to verify that the quantity $\xi(v)$ is invariant under diffeomorphism group transformations operating from the right, i.e.,

$$
\xi(v) \rightarrow \xi(v), \quad f(u) \rightarrow f(\alpha(u))
$$

but that it transforms as a density of weight -1 under the inverse diffeomorphism group transformations operating from the left, i.e.,

$$
\xi(w) \rightarrow \frac{1}{\left(\frac{d \beta^{-1}(w)}{d w}\right)} \xi\left(\beta^{-1}(w)\right), \quad f(u) \rightarrow \beta(f(u)) \equiv w .
$$

The only quadratic form in $\xi$ that is invariant under both of these two transformations is [2]

$$
\langle\xi, \xi\rangle \equiv \int_{0}^{1} \tau d v(\tau \xi)^{2}(v)
$$

provided that $\tau$ remains invariant under the first transformation, but

$$
\tau \rightarrow \tau\left(\frac{d \beta^{-1}(w)}{d w}\right)
$$

under the second. The geometric meaning of these transformations is not difficult to visualize. In the case of (2.22), $N(u)$ as parameterized by (2.16) transforms according to Eq. (2.2) under right multiplication of the fiber coordinate $f \rightarrow f \circ \alpha$, while the coordinate in the quotient space $\mathcal{M} / \mathcal{G}, \tau$ remains invariant. In the second transformation (2.23), the base space coordinate $\tau$ transforms according to (2.25), in order to cancel the change under left multiplication, $f \rightarrow \beta \circ f$ and leave the total $N(u)$ invariant.

With the fully invariant inner product on the cotangent space to $\mathcal{G}$ defined by Eq. (2.24), we may define the integration measure on $\mathcal{G}$ by the Gaussian normalization condition,

$$
\int[\mathcal{D} \xi] \exp \left\{-\frac{i}{2}\langle\xi, \xi\rangle\right\}=1
$$

Returning then to our problem of evaluating the Jacobian $J$ in Eq. (2.17), we find that

$$
\langle\delta N, \delta N\rangle_{N}=\frac{(\delta \tau)^{2}}{\tau}+\frac{1}{\tau^{2}}\left\langle\xi, \Delta_{0} \xi\right\rangle
$$

with $\Delta_{0}$ given by the same second order operator (with $\omega=0$ ) whose determinant we evaluated in the previous section. Therefore we have immediately, 


$$
\begin{aligned}
1 & =\int[\mathcal{D}(\delta N)] \exp \left\{-\frac{i}{2}\langle\delta N, \delta N\rangle_{N}\right\} \\
& =\int d(\delta \tau) J(\tau) \int[\mathcal{D} \xi] \exp \left\{-\frac{i(\delta \tau)^{2}}{2 \tau}-\frac{i\left\langle\xi, \Delta_{0} \xi\right\rangle}{2 \tau^{2}}\right\} \\
& =J\left(\frac{2 \pi \tau}{i}\right)^{\frac{1}{2}}\left[\operatorname{det}\left(\frac{\Delta_{0}}{\tau^{2}}\right)\right]^{-\frac{1}{2}} \\
& =\text { const. } \times J
\end{aligned}
$$

where the last result follows by Eq. (1.23). Hence the Jacobian $J$ is a constant independent of $\tau$.

The evaluation of the propagator in Eq. (2.18) is now straightforward. The functional integration over $x^{a}(u)$ with fixed enpoint conditions is the same as that for the free nonrelativistic particle, and we are left with only a simple integral over the proper time $\tau$ to perform:

$$
\begin{aligned}
G\left(x, x_{0}\right) & =J \int_{0}^{\infty} d \tau(2 \pi i \tau)^{-\frac{D}{2}} \exp \left\{\frac{i}{2 \tau}\left(x-x_{0}\right)^{a} \eta_{a b}\left(x-x_{0}\right)^{b}-\frac{i}{2} \tau m^{2}\right\} \\
& =J \int_{0}^{\infty} d \tau \int \frac{d^{D} p}{(2 \pi)^{D}} \exp \left\{i p_{a}\left(x-x_{0}\right)^{a}-\frac{i \tau}{2}\left(p_{a} \eta^{a b} p_{b}+m^{2}\right)\right\} \\
& =\int \frac{d^{D} p}{(2 \pi)^{D}} \frac{e^{i p \cdot\left(x-x_{0}\right)}}{\left(p^{2}+m^{2}-i 0\right)},
\end{aligned}
$$

provided that the constant $J=\frac{i}{2}$ and an infinitesimal negative imaginary part is added to $m^{2}$ to define the $\tau$ integral. With this normalization Eq. (2.29) is recognized as just the Feynman propagator for the free relativistic scalar field, which we have obtained here by the path integral treatment of the reparameterization invariant first quantized particle action (2.1). As in the non-relativistic case, the arbitrary multiplicative constant is the analog of a constant of integration which can only be fixed by the normalization condition on $G$.

\section{B. Non-Abelian Gauge Theory}

The Yang-Mills action,

$$
S_{i n v}[A]=-\frac{1}{4 g^{2}} \int d^{4} x F_{\mu \nu}^{i} F^{i \mu \nu}
$$

is invariant under non-abelian gauge transformations, $A_{\mu}^{i} \rightarrow B_{\mu}$ with $A$ and $B$ related by 


$$
A_{\mu}^{i}=\left\{\mathcal{U}^{-1}(\theta) B_{\mu} \mathcal{U}(\theta)\right\}^{i}-i\left\{\mathcal{U}^{-1}(\theta) \partial_{\mu} \mathcal{U}(\theta)\right\}^{i}
$$

and $\mathcal{U}$ is an arbitrary element of the gauge group at each spacetime point. Thus, $\mathcal{U}$ generates the class of field configurations gauge equivalent to $B_{\mu}^{i}$. For $\mathcal{U}$ close to the identity the infinitesimal form of Eq. (2.31) is:

$$
A_{\mu}^{i} \rightarrow A_{\mu}^{i(\theta)} \equiv A_{\mu}^{i}+\left(\nabla_{\mu} \theta\right)^{i} \equiv A_{\mu}^{i}+\partial_{\mu} \theta^{i}+f^{i j k} A_{\mu}^{j} \theta^{k},
$$

where $f^{i j k}$ are the structure constants of some non-abelian gauge group.

We may regard Eq. (2.31) as setting up a coordinate system in the space of all gauge fields $\mathcal{M}$ with gauge fiber coordinates on $\mathcal{G}$ specified by $\mathcal{U}(\theta)$ and base space coordinates in the quotient space $\mathcal{M} / \mathcal{G}$ specified by $B_{\mu}^{i}$, analogous to (2.16) of the relativistic point particle example. Then we consider the only Poincare invariant quadratic inner product on the cotangent space, namely

$$
\langle\delta A, \delta A\rangle=\int d^{4} x \delta A_{\mu}^{i}(x) \eta^{\mu \nu} \delta A_{\nu}^{i}(x) .
$$

This natural, invariant quadratic form is completely analogous to the invariant line element $d s^{2}$ of finite dimensional Riemmanian manifolds, and therefore induces a natural, invariant volume form on the space of gauge fields. In the present case this metric is flat, being independent of $A_{\mu}^{i}(x)$. Hence the invariant volume form is just the product of coordinate differentials. We may fix the normalization of this functional measure by a Gaussian normalization condition:

$$
\int\left[\mathcal{D} \delta A_{\mu}^{i}\right] \exp \left(-\frac{i}{2}\langle\delta A, \delta A\rangle\right)=1
$$

The measure, defined in this way is manifestly Lorentz invariant. Moreover, it is gauge invariant under (2.32). This is because it is translational invariant under $\delta A_{\mu}^{i}(x) \rightarrow \delta A_{\mu}^{i}(x)+$ $v_{\mu}^{i}(x)$, and invariant under global gauge rotations. Let us now write

$$
A_{\mu}^{i}=\bar{A}_{\mu}^{i}+a_{\mu}^{i}
$$

where $\bar{A}_{\mu}^{i}$ is a fixed background Yang-Mills field and $a_{\mu}^{i}$ is the variable of integration. The corresponding $B_{\mu}^{i}$ is given by 


$$
B_{\mu}^{i}=\bar{B}_{\mu}^{i}+b_{\mu}^{i}=\bar{A}_{\mu}^{i}+b_{\mu}^{i}
$$

where we have used the arbitrariness in the separation of background field and and integration variable to set $\bar{B}=\bar{A}$. Then the path integral

$$
Z[\bar{A}]=(\operatorname{Vol}(\mathcal{G}))^{-1} \int\left[\mathcal{D} a_{\mu}^{i}\right] \exp \left(i S_{i n v}[\bar{A}+b]\right)
$$

may be defined with respect to the measure induced by the definitions (2.33) and (2.34). Here $\operatorname{Vol}(\mathcal{G})$ denotes schematically the (infinite) gauge orbit volume that must be divided out of $\left[\mathcal{D} a_{\mu}^{i}\right.$, and $b$ denotes the integration variable over the equivalence classes of potentials in $\mathcal{M} / \mathcal{G}$. In order to specify the field coordinates completely one still needs to fix a condition on the $b_{\mu}^{i}$ of the form,

$$
(F \cdot b)^{i}=0
$$

For example $F$ might be the covariant derivative with respect to the background gauge potential $\bar{A}$, in which case the results will be formally identical to those obtained in the background field Landau gauge. However, it is important to emphasize that in the geometric approach follwed here this is not a gauge "fixing" that breaks the gauge invariance of the theory, but rather merely a particular choice of coordinates in a manifestly coordinate (i.e. gauge) invariant construction, since $b_{\mu}^{i}$ lies by definition in the quotient space $\mathcal{M} / \mathcal{G}$.

Again, our task is to express the gauge invariant quantity (2.37) in the coordinates (or gauge) specified by (2.31) and (2.38). We first compute the Jacobian of the transformation to the new coordinates

$$
\left[\mathcal{D} a_{\mu}^{i}\right]=J\left[\mathcal{D} b_{\mu}^{i}\right]\left[\mathcal{D} \theta^{i}\right]
$$

in the tangent space:

$$
\begin{aligned}
1 & =\int\left[\mathcal{D} a_{\mu}^{i}\right] \exp \left(-\frac{i}{2}\langle a, a\rangle\right) \\
& =\int J\left[\mathcal{D} b_{\mu}^{i}\right]\left[\mathcal{D} \theta^{i}\right] \exp \left(-\frac{i}{2}(\langle b, b\rangle+2\langle\nabla \theta, b\rangle+\langle\nabla \theta, \nabla \theta\rangle)\right)
\end{aligned}
$$


This integral may be computed by completing the square and using condition (2.38). We find that

$$
J=\left[\left.\operatorname{det}_{S}\left(-\nabla^{2}\right) \operatorname{det}_{V}\left(\delta_{\mu}^{\nu}-\nabla_{\mu}\left(\nabla^{2}\right)^{-1} \nabla^{\nu}\right)\right|_{F}\right]^{\frac{1}{2}}
$$

where the vector determinant is to be evaluated over the space of fields $b_{\nu}^{i}$ obeying the condition (2.38). This vector determinant may be converted into a scalar determinant [4] and the Jacobian (2.41) is given finally by:

$$
J=\operatorname{det}_{S}^{-\frac{1}{2}}\left(F \cdot F^{\dagger}\right) \operatorname{det}_{S}(F \cdot \nabla)
$$

where $F^{\dagger}$ is the adjoint of $F$ with respect to the inner product (2.33). In this form connection to the standard gauge-fixed perturbative formalulation of Yang-Mills theory may be made, since the second factor in (2.42) is precisely the Fadeev-Popov Jacobian $\Delta_{F P}$ of the gauge fixing method for the gauge condition (2.38), while the first factor is a constant, independent of the field point $A_{\mu}$, and therefore may be taken out of the path integral, without affecting the result. It is important to retain this factor if one calculates the effective action as a function of the background field, however.

Having determined the correct Jacobian of the transformation to field coordinates, $\left(b_{\mu}, \theta\right)$, we may now express $Z$ in the form,

$$
\begin{aligned}
Z[\bar{A}] & =(\operatorname{Vol}(\mathcal{G}))^{-1} \int\left[\mathcal{D} \theta^{i}\right] \int J\left[\mathcal{D} b_{\mu}^{i}\right] \exp \left(i S_{\text {inv }}[\bar{A}+b]\right) \\
& =\left.\operatorname{det}_{S}^{-\frac{1}{2}}\left(-\bar{\nabla}^{2}\right) \int\left[\mathcal{D} b_{\mu}^{i}\right] \operatorname{det}_{S}(-\bar{\nabla} \cdot \nabla)\right|_{A=\bar{A}+b} \exp \left(i S_{i n v}[\bar{A}+b]\right),
\end{aligned}
$$

where the backgound Landau gauge $F^{\mu}=\bar{\nabla}^{\mu}$ has been chosen and the integral is over the gauge invariant field coordinate, $b_{\mu}^{i}$. The gauge volume factor has been cancelled explicitly. If one expands the invariant action in a power series in the integration variable $b$, treating the quadratic Gaussian functional integral exactly, but the higher powers of $b$ as perturbations, it is clear that this form generates the same Feynman rules as the gauge fixed path integral of more standard formulations, and that a similar equivalence holds for other choices for the gauge fixing function $F$. In the present approach, the geometric significance of gauge 
fixing as simply a choice of coordinates in a coordinate invariant expression is manifest and proofs of gauge invariance are unnecessary. Moreover, the non-perturbative aspects of the

path integral and the correct dependence on the background field $\bar{A}$ in $\operatorname{det}{ }_{S}^{-\frac{1}{2}}\left(-\bar{\nabla}^{2}\right)$ has been obtained as well. This background field dependence is essential for the proof of Ward identities in the background field method, and may be lost in a careless treatment of the gauge-fixing. The background field method is an extremely efficient method of extracting physical results, such as asymptotic freedom in non-abelian gauge theories, with a minimum of technical complication. Provided the functional determinants in these formal expressions can be defined by a suitable invariant regulator, such as the $\zeta$-function method of the last section, (2.43) defines the configuration space path integral for Yang-Mills theory in a manifestly gauge and Lorentz invariant manner without recourse to discretization and limiting procedures.

One possible problem that this construction of the functional integral for non-abelian gauge fields glosses over is the question of a global choice of coordinates on the coset space. The construction assumes that we can choose coordinates on the full configuration space such that the slice specified by (2.38) in the coset space $\frac{\mathcal{M}}{\mathcal{G}}$ intersects each gauge fiber $\mathcal{G}$ once and only once. This is by no means guaranteed in general, and in fact, it is known that for certain common choices of $F$ this is not the case [13. An interesting open issue is whether or not it is possible to find a global choice of coordinates on the configuration space that does satisfy this criterion.

\section{The Partition Function for a Finite Temperature Photon Gas}

As a simple application of the geometric path integral in the case of a theory with an internal gauge group, let us consider how one would calculate the partition function and thermodynamics of a gas of free photons. If we set the background field $\bar{A}=0$ in the expression (2.43), and continue to imaginary time, $t \rightarrow i t_{E}, i S_{i n v} \rightarrow-S_{E}$ we obtain the formal result for the partition function $Z$ for a general Yang-Mills theory with action (2.30). 
If we take the gauge group to be the abelian group $U(1)$ of electromagnetism, so that the structure constants $f_{i j k}=0$ as well, then $\nabla=\bar{\nabla}=\partial$ and $Z$ reduces to

$$
Z=\operatorname{det}_{S}^{+\frac{1}{2}}(-\square) \int\left[\mathcal{D} b_{\mu}^{i}\right] \exp \left(-S_{E}[b]\right)
$$

where the Euclidean action may be written in the form,

$$
S_{E}[b]=+\frac{1}{4 g^{2}} \int d^{4} x_{E} F_{\mu \nu} F^{\mu \nu}=\frac{1}{2 g^{2}} \int d^{4} x_{E} b_{\mu}(-\square) b_{\mu}
$$

in the Landau gauge $F \cdot b=\partial_{\mu} b_{\mu}=0$. Then the Gaussian path integral (2.44) is immediately performed to yield

$$
Z=\left.\operatorname{det}_{S}^{+\frac{1}{2}}(-\square) \operatorname{det}_{V}^{-\frac{1}{2}}(-\square)\right|_{F},
$$

where the vector determinant is to be evaluated over vectors satisfying the Landau transversality condition. Since any four vector may be decomposed into transverse and longitudinal components which decomposition is orthogonal with respect to the inner product (2.33), we may extend the evaluation of the vector determinant over all four vectors provided we divide out the longitudinal contribution, i.e.

$$
\left.\operatorname{det}_{V}(-\square)\right|_{F}=\operatorname{det}_{V}(-\square) / \operatorname{det}_{S}(-\square)=\operatorname{det}_{S}^{3}(-\square)
$$

where the last step follows by introducing a Cartesian basis for the four vectors, so that the unrestricted vector determinant is just the determinant of the scalar operator $-\square$ raised to the fourth power. Hence,

$$
Z=\operatorname{det}_{S}^{-1}(-\square)
$$

which is the same as the result for two free massless scalar fields. These are the two physical helicity states of a massless photon (or gluon). In this form the significance of the Jacobian factor (2.42) may be understood. Its role is precisely to cancel unphysical degrees of freedom in the covariant four vector gauge field, reducing the contribution to just the correct physical degrees of freedom of the canonical method of quantization. 
To complete the evaluation of the partition function for the photon gas we have only to note that finite temperature implies that the operator $-\square$ should be evaluated over functions periodic in Euclidean time with period $\beta$ equal to the inverse temperature $T^{-1}$. Hence its eigenvalues are just

$$
\left(\frac{2 \pi n}{\beta}\right)^{2}+\vec{k}^{2}
$$

and the result for $Z$ in (2.48) is nothing but the square of the product over wavenumbers $\vec{k}$ of the harmonic oscillator result (1.25) with the frequency $\omega$ replaced by $\omega_{k}=|\vec{k}|$, i.e.

$$
Z \equiv e^{-\beta F}=\left[\prod_{\vec{k}} \frac{1}{2 \sinh \left(\frac{\omega_{k} \beta}{2}\right)}\right]^{2}
$$

so that the free energy function is

$$
F=-T \ln Z=2 T \sum_{\vec{k}} \ln \left(e^{\frac{\omega_{k} \beta}{2}}-e^{-\frac{\omega_{k} \beta}{2}}\right)=\sum_{\vec{k}} \omega_{k}+2 T \sum_{\vec{k}} \ln \left(1-e^{-\omega_{k} \beta}\right),
$$

the first term of which is the infinite, but temperature independent sum of zero point energies $\frac{\omega_{k}}{2}$ for each of the two photon helicity states. This ultraviolet quartic divergence in the zero temperature free energy is a general feature of field theory, and leads to the "cosmological constant problem" when gravitation is considered, since there are no observable effects of this zero-point energy on the curvature of spacetime. This divergence would be fully regulated by the $\zeta$-function method if we performed the sum over $\vec{k}$ (as well as $n$ ) before analytic continuation to $s=0$, although this involves a more difficult calculation. In any case, the question of the absolute zero point of free energy does not arise if we are interested only in energy differences or the temperature dependence of the free energy, which is given entirely by the second term of eq. (2.51) above. Taking the infinite volume continuum limit so that we may replace the sum over $\vec{k}$ by an integral, and dividing by the volume, we find the usual result for the free energy density of the photon gas, viz.

$$
2 T \int \frac{d^{3} \vec{k}}{(2 \pi)^{3}} \ln \left(1-e^{-\omega_{k} \beta}\right)=-\frac{\pi^{2}}{45} T^{4},
$$

from which all the other usual thermodynamic relations follow. If we had considered YangMills theory with gauge group $S U(N)$ then this same result holds to lowest order in the 
coupling $g$ if multiplied by the number of independent fields in the adjoint representation of $S U(N)$, namely $N^{2}-1$. This is the leading contribution to the free energy density of gluons in QCD (where $N=3$ ) at high temperature.

Thus, the covariant, geometrically constructed path integral of the last section, together with a covariant definition of the functional determinants by means of the $\zeta$-function, yields the same result for the finite temperature free photon or gluon gas as that obtained by the more standard (but non-covariant) canonical quantization in Fock space. The Bose-Einstein statistics of the logarithm in (2.51) comes out of the covariant treatment automatically and does not require any additional assumptions. One can treat fermionic degrees of freedom analogously by the introduction of anti-commuting Grassman variables into the path integral, and obtain Fermi-Dirac statistics for the corresponding thermodynamic functions of a gas of spin- $\frac{1}{2}$ particles. These results may be obtained very rapidly within the covariant path integral framework.

\section{THE FUNCTIONAL INTEGRAL OVER GEOMETRIES}

In the path integral approach to quantizing gravity a very important technical issue that must be faced is the correct specification of the functional measure on the coset space of spacetime manifolds modulo coordinate reparameterizations. Although this problem has been solved, originally by following the algebraic method based on the Becchi-Rouet-StoraTyutin (BRST) global supersymmetry of the gauge fixed path integral [14], the issue of the functional measure in the path integral for quantum gravity has continued to be a source of confusion. Accordingly, it is worthwhile to rederive the BRST result by the more intuitive geometric method of constructing the invariant volume element on the cotangent space of metrics. The two methods lead to the same result [4].

One begins by recognizing that the action of classical general relativity (or more generally of any theory consistent with the principle of equivalence) is invariant under general coordinate transformations, $x^{\mu} \rightarrow X^{\mu}=X^{\mu}(x)$ of the manifold of spacetime. The infinitesimal 
form of this transformation is

$$
x^{\mu} \rightarrow x^{\mu}+\xi^{\mu}(x) .
$$

We shall construct the functional measure in the path integral for quantum gravity by requiring that it also be invariant under (3.1), following the same strategy as in the previous examples [⿴囗十 . Treat an arbitrary spacetime metric $g_{\mu \nu}(x)$ as the coordinate of a "point" in the function space of all metrics, denoted by $\mathcal{M}$. The infinitesimal one-form, $\delta g_{\mu \nu}(x) \equiv h_{\mu \nu}(x)$ lies in the cotangent space to $\mathcal{M}$ at the point $g_{\mu \nu}(x)$. We define a quadratic inner product on this cotangent space of one-forms by

$$
\langle h, h\rangle_{T} \equiv \int d^{4} x \sqrt{-g} h_{\mu \nu}(x) G^{\mu \nu \rho \sigma} h_{\rho \sigma}(x)
$$

The subscript $T$ reminds us that this is an inner product for tensors. Now the scalar $d s^{2}=g_{\mu \nu}(x) d x^{\mu} d x^{\nu}$ is invariant under the (passive) relabelling of the coordinates of spacetime, (3.1) that leaves the geometric spacetime point unchanged. The corresponding transformation of the metric on the spacetime manifold is

$$
h_{\mu \nu}(x) \rightarrow h_{\mu \nu}(x)+\nabla_{\mu} \xi_{\nu}+\nabla_{\nu} \xi_{\mu}
$$

This may now be regarded as a relabelling of coordinates on $\mathcal{M}$ which leaves the point, i.e. the geometry corresponding to $g_{\mu \nu}(x)$ unchanged. Hence we must require that the measure be invariant under the transformations (3.1) and (3.3) as well. Since $h_{\mu \nu}(x)$ transforms covariantly as a symmetric tensor under (3.1), $G^{\mu \nu \rho \sigma}$ must transform like a contravariant four-tensor. Like $g_{\mu \nu}(x), G^{\mu \nu \rho \sigma}(g)$ has evident symmetry properties: it is symmetric under interchange of its first two indices or its last two indices, as well as interchange of the first two with the last two. Finally, again like $g_{\mu \nu}(x), G^{\mu \nu \rho \sigma}(g)$ must be a purely local function of the coordinates of $\mathcal{M}$. That is, it should contain no derivatives of $g_{\mu \nu}(x)$. The unique ultralocal tensors with these properties are [16]

$$
\frac{1}{2}\left(g^{\mu \rho} g^{\nu \sigma}+g^{\mu \sigma} g^{\nu \rho}\right) \quad \text { and } \quad g^{\mu \nu} g^{\rho \sigma}
$$


If we did not demand ultralocality, an infinite number of tensors involving higher derivatives would appear in this list. Using such tensors in the definition of the inner product and functional measure ultimately would have the effect of defining a different set of dynamical coordinates for the theory. Since we assume that the metric is the fundamental field coordinate, and derivatives of it in the action introduce dynamics, we do not wish to introduce derivatives and spurious dynamics into the essentially kinematic definition of the inner product or functional measure. In fact, this is the only principle which justifies an otherwise quite arbitrary distinguishing of the functional measure in the path integral from the action functional.

Restricting the metric on $\mathcal{M}$ to be covariant and ultralocal determines it (up to an overall irrelevant normalization) to be:

$$
G^{\mu \nu \rho \sigma}=\frac{1}{2}\left(g^{\mu \rho} g^{\nu \sigma}+g^{\mu \sigma} g^{\nu \rho}+C g^{\mu \nu} g^{\rho \sigma}\right)
$$

where $C$ is an undetermined constant.

Having endowed the function space of metrics $\mathcal{M}$ itself with a metric, (the "supermetric" in the language of De Witt [16]) we are now in a position to define an invariant volume form on the space. In order to avoid confusion it is useful to introduce the vielbein field, $e_{\mu}^{m}$ which converts spacetime vector indices to spacetime tangent space indices. If we then define the density,

$$
\tilde{h}_{m n} \equiv \sqrt{e} e_{m}^{\mu} e_{n}^{\nu} h_{\mu \nu}
$$

together with the relations,

$$
g_{\mu \nu}=e_{\mu}^{m} e_{\nu}^{n} \eta_{m n}
$$

and

$$
e \equiv \operatorname{det}\left(e_{\mu}^{m}\right)=\sqrt{-g},
$$

then the inner product (3.2) may be expressed in terms of the flat spacetime metric, $\eta_{m n}=$ $\operatorname{diag}(-1,1,1,1)$ as 


$$
\langle h, h\rangle_{T}=\int d^{4} x \tilde{h}_{m n} \tilde{G}^{m n r s} \tilde{h}_{r s}
$$

where

$$
\tilde{G}^{m n r s}=\frac{1}{2}\left(\eta^{m r} \eta^{n s}+\eta^{m s} \eta^{n r}+C \eta^{m n} \eta^{r s}\right)
$$

is independent of $x$.

Then by analogy with the invariant volume form on a pseudo-Riemannian manifold, $\prod_{m} d \tilde{x}^{m}=\operatorname{det}\left(e_{\mu}^{m}\right) \prod_{\mu} d x^{\mu}$, we would like to define the invariant volume form on the function space of metrics $\mathcal{M}$ by:

$$
\left[\mathcal{D} h_{\mu \nu}\right] \equiv \text { const. } \times \prod_{x, m \leq n} d \tilde{h}_{m n}(x)=\text { const. } \times \prod_{x} e^{\frac{(D-4)(D+1)}{4}} \prod_{\mu \leq \nu} d h_{\mu \nu}(x)
$$

in $D$ spacetime dimensions. Although useful for some formal manipulations this "definition" leads to ambiguities for continuum functional integrals, because of the ill-defined nature of the product at each spacetime point, which also leaves the normalization of the measure undefined. One would again have to resort to some kind of discretization and limiting procedure in order to give meaning to (3.11). More than just a technical inconvenience such a procedure would be in grave danger of violating general covariance by imposing a rigid skeletonization on a dynamical theory of spacetime, which should have no a priori preferred geometry inherent in its definition. Instead, as we have seen in the previous examples, it is preferable to define the measure by the Gaussian normalization condition,

$$
\int\left[\mathcal{D} h_{\mu \nu}\right] \exp \left(-\frac{i}{2}\langle h, h\rangle_{T}\right)=1
$$

which is formally satisfied by (3.11).

The overall constant in front of the supermetric, (3.5) is irrelevant, since it may always be reabsorbed into the normalization integral, (3.12). The constant $C$ is not irrelevant, since it determines the signature of the supermetric on $\mathcal{M}$. This may be seen by decomposing the arbitrary tangent space tensor into its tracefree and trace parts,

$$
h_{\mu \nu}=h_{\mu \nu}^{T F}+\frac{h g_{\mu \nu}}{4}
$$


and operating on this decomposition with the supermetric $G$. The $\frac{(D+2)(D-1)}{2}$ tracefree parts of $h_{\mu \nu}$ in $D$ dimensions are mapped onto $h^{T F \mu \nu}$, independent of $C$. However, on the scalar trace mode $G$ has eigenvalue, $1+\frac{C D}{2}$. Hence the signature of $G$ depends on the value of $C$ : for $C>-\frac{D}{2}$, the signature of $G$ in the scalar trace sector is positive, while for $C<-\frac{D}{2}$ the signature is negative. If $C=-\frac{D}{2}$, the metric is non-invertible and becomes a projector onto the tracefree subspace. For the moment let us leave $C$ undetermined.

For any value of $C$ it is clear that the functional measure defined with reference to the inner product, $\langle h, h\rangle_{T}$ is invariant under the infinitesimal general coordinate transformation, (3.1). Once we have a coordinate invariant functional measure, we must extract the infinite gauge orbit volume in an invariant way as well. To this end, we introduce a change of coordinates in the tangent space of $\mathcal{M}$ at $g_{\mu \nu}$ :

$$
h_{\mu \nu}=h_{\mu \nu}^{\perp}+(L \xi)_{\mu \nu}+\left(2 \sigma+\frac{2}{D} \nabla_{\lambda} \xi^{\lambda}\right) g_{\mu \nu}
$$

where $L$, (the "conformal Killing form") maps vectors into traceless symmetric tensors,

$$
(L \xi)_{\mu \nu} \equiv \nabla_{\mu} \xi_{\nu}+\nabla_{\mu} \xi_{\nu}-\frac{2}{D}\left(\nabla_{\lambda} \xi^{\lambda}\right) g_{\mu \nu}
$$

Thus, $L \xi$ spans all symmetric tensors which are gauge transforms of $h_{\mu \nu}^{T F}$, the traceless part of $h_{\mu \nu}$. The scalar $\sigma$ is the gauge invariant piece of the trace, and $h_{\mu \nu}^{\perp}$ is the gauge invariant piece of $h_{\mu \nu}^{T F}$. Now $h_{\mu \nu}^{\perp}$ may be chosen to lie in the orthogonal complement to $L$, with respect to the inner product (3.2), which requires $\left(L^{\dagger} h^{\perp}\right)_{\mu}=-2 \nabla^{\nu} h_{\mu \nu}^{\perp}=0$. Indeed this is the simplest choice for doing one-loop calculations, [4] which involve only Gaussian integrals, and justifies the notation $h^{\perp}$. However, the choice of orthogonal coordinates on the tangent space of $\mathcal{M}$ is by no means necessary, and $h_{\mu \nu}^{\perp}$ may be required to satisfy an arbitrary coordinate (gauge) condition:

$$
\left(F \cdot h^{\perp}\right)_{\mu} \equiv F^{\nu} h_{\mu \nu}^{\perp}=0 .
$$

The only condition on $F$ is that the operator $F \circ L$ be locally invertible, so that (3.14) can be solved uniquely for $\xi$ : 


$$
\xi_{\mu}=(F \circ L)_{\mu}^{-1 \nu}\left(F \circ h^{T F}\right)_{\nu}
$$

Otherwise, the local coordinate chart, (3.14) is singular at the point $g_{\mu \nu}$.

Following the discussion in the previous section for the case of the non-abelian gauge field, to extract the infinite gauge orbit volume generated by the gauge direction, $\xi_{\mu}$ we must find the Jacobian of the transformation to the new field coordinates, $\left(h_{\mu \nu}^{\perp}, \xi_{\mu}, \sigma\right)$ :

$$
\left[\mathcal{D} h_{\mu \nu}\right]=J\left[\mathcal{D} h_{\mu \nu}^{\perp}\right]\left[\mathcal{D} \xi_{\mu}\right][\mathcal{D} \sigma]
$$

This is accomplished by substituting the decomposition, (3.14) into the inner product (3.2), completing the square of the term quadratic in $\xi_{\mu}$, and computing the Gaussian integrals over each of the components respectively:

$$
\begin{aligned}
1=\int\left[\mathcal{D} h_{\mu \nu}\right] \exp \left(-\frac{i}{2}\langle h, h\rangle_{T}\right) \\
=J \int\left[\mathcal{D} h_{\mu \nu}^{\perp}\right] \exp \left(-\frac{i}{2}\left\langle h^{\perp},(1-M) h^{\perp}\right\rangle_{T}\right) \int\left[\mathcal{D} \xi_{\mu}\right] \exp \left(-\frac{i}{2}\left\langle\xi^{\prime}, \Delta_{1} \xi^{\prime}\right\rangle_{V}\right) \\
\times \int[\mathcal{D} \sigma] \exp \left(-8 i(1+2 C)\langle\sigma, \sigma\rangle_{S}\right),
\end{aligned}
$$

where the vector Laplacian $\Delta_{1}$ is defined by:

$$
\left(\Delta_{1}\right)_{\mu}^{\nu} \equiv\left(L^{\dagger} L\right)_{\mu}^{\nu}=-2\left(\delta_{\mu}^{\nu} \nabla^{2}+\left(1-\frac{2}{D}\right) \nabla_{\mu} \nabla^{\nu}+R_{\mu}^{\nu}\right)
$$

the tensor operator $M$ is given by:

$$
M_{\mu \nu}{ }^{\rho \sigma} \equiv\left(L\left(\Delta_{1}\right)^{-1} L^{\dagger}\right)_{\mu \nu}{ }^{\rho \sigma}=-2 \nabla_{\mu}\left(\Delta_{1}^{-1}\right)_{\nu}{ }^{\rho} \nabla^{\sigma}-2 \nabla_{\nu}\left(\Delta_{1}^{-1}\right)_{\mu}{ }^{\rho} \nabla^{\sigma}+g_{\mu \nu} \nabla^{\lambda}\left(\Delta_{1}^{-1}\right)_{\lambda}{ }^{\rho} \nabla^{\sigma}
$$

and $\xi_{\mu}^{\prime}=\xi_{\mu}+\left(\Delta_{1}^{-1} L^{\dagger} h^{\perp}\right)_{\mu}$ is the shifted vector obtained by completing the square. The notations $\langle,\rangle_{V}$ and $\langle,\rangle_{S}$ denote the covariant inner products on vectors and scalars respectively:

$$
\begin{gathered}
\langle\xi, \xi\rangle_{V}=\int d^{4} x \sqrt{-g} \xi_{\mu} g^{\mu \nu} \xi_{\nu}, \\
\langle\sigma, \sigma\rangle_{S}=\int d^{4} x \sqrt{-g} \sigma^{2}
\end{gathered}
$$


The remaining tensor and vector Gaussian functional integrals in (3.19) can be simplified [4] to yield finally the result for the Jacobian:

$$
J=\left[\left.\operatorname{det}_{T}(1-M)\right|_{F}\right]^{\frac{1}{2}}\left[\operatorname{det}_{V} \Delta_{1}\right]^{\frac{1}{2}}=\left[\operatorname{det}_{V}\left(F \circ F^{\dagger}\right)\right]^{-\frac{1}{2}} \operatorname{det}_{V}(F \circ L),
$$

in complete analogy with the gauge theory result (2.42). The second factor will be recognized as the Fadeev-Popov determinant for the gauge (3.16) on the tracefree components of $h_{\mu \nu}$, while the first factor is a $h_{\mu \nu}$-independent normalization factor that makes no contribution to the Feynman rules. Notice that the Jacobian factor has been derived by tangent space methods, involving only Gaussian functional integrals, but that this involves no restriction to one-loop order. The result (3.24) is valid to all orders of perturbation theory.

With the Jacobian (3.24), we now know how to factor the infinite diffeomorphism gauge group volume out of the covariant quantum measure, (3.18) in a manifestly covariant way. If the action is independent of the vector gauge orbit parameter $\xi_{\mu}$, integration over $\xi_{\mu}$ would simply yield the infinite volume of the diffeomorphism group, so

$$
\begin{aligned}
\{\operatorname{Vol}(\mathcal{G})\}^{-1} \int\left[\mathcal{D} h_{\mu \nu}\right] & =\{\operatorname{Vol}(\mathcal{G})\}^{-1} \int\left[\mathcal{D} \xi_{\mu}\right] \int J\left[\mathcal{D} h_{\mu \nu}^{\perp}\right][\mathcal{D} \sigma] \\
& =\int J\left[\mathcal{D} h_{\mu \nu}^{\perp}\right][\mathcal{D} \sigma]
\end{aligned}
$$

when integrated over functions independent of $\xi_{\mu}$.

The final steps in constructing the covariant path integral for quantum gravity involves extending the integration measure defined on the tangent space to a measure on the full metric. The extension of the coordinates on the tangent space (3.14) to coordinates on $\mathcal{M}$ is straightforward, at least locally. We write

$$
\begin{aligned}
g_{\mu \nu}(x) & =\frac{\partial X^{\rho}}{\partial x^{\mu}} \frac{\partial X^{\sigma}}{\partial x^{\nu}} e^{2 \sigma(X)} g_{\rho \sigma}^{\perp}(X), \\
\left(F \cdot g^{\perp}\right)_{\mu} & =0 .
\end{aligned}
$$

where $\sigma$ may be fixed by the requirement that $g^{\perp}$ has constant scalar curvature $\left(R^{\perp}=\right.$ const.):

$$
R\left[g_{\mu \nu}\right]=6 e^{-3 \sigma} \Delta_{0}^{\perp} e^{\sigma}+e^{2 \sigma} R^{\perp}
$$


and $\Delta_{0}^{\perp}$ is the scalar Laplace-Beltrami operator with respect to the metric $g^{\perp}$. The vacuum amplitude for quantum gravity may then be written in the succint generally covariant form:

$$
\begin{aligned}
Z & =\{\operatorname{Vol}(\mathcal{G})\}^{-1} \int\left[\mathcal{D} g_{\mu \nu}\right] \exp \left(i S_{i n v}[g]\right) \\
& =\int J\left[\mathcal{D} g_{\mu \nu}^{\perp}\right][\mathcal{D} \sigma] \exp \left(i S_{i n v}\left[e^{2 \sigma} g^{\perp}\right]\right) \\
& =\left.\left[\operatorname{det}_{V}\left(F \circ F^{\dagger}\right)\right]^{-\frac{1}{2}} \int[\mathcal{D} \sigma]\left[\mathcal{D} g_{\mu \nu}^{\perp}\right] \operatorname{det}_{V}(F \circ L)\right|_{g=e^{2 \sigma} g^{\perp}} \exp \left(i S_{i n v}\left[e^{2 \sigma} g^{\perp}\right]\right),
\end{aligned}
$$

where (3.24) has been used. This, together with Eqs. (3.15), (3.26), and (3.27) is the final result for the invariant functional integral over geometries.

Note that the infinite volume of the non-compact group of spacetime coordinate transformations does not appear in (3.28), and that the functional measure in the invariant integration over $\sigma$ and $g^{\perp}$ is determined completely by the Gaussian normalization condition (3.12) on the cotangent space in the decomposition (3.14).

If we set

$$
g_{\mu \nu}^{\perp}=\bar{g}_{\mu \nu}+h_{\mu \nu}^{\perp},
$$

the analogy with the gauge theory result (2.43) will be evident. In both cases the $a^{\perp}$ or $h^{\perp}$ independent determinant makes no contribution to the Feynman rules. It arises only because

$$
\left[\mathcal{D} h_{\mu \nu}\right] \delta(F \cdot h)=\left[\operatorname{det}_{V}\left(F \circ F^{\dagger}\right)\right]^{-\frac{1}{2}}\left[\mathcal{D} h_{\mu \nu}^{\perp}\right]
$$

when both the $\left[\mathcal{D} h_{\mu \nu}\right]$ and $\left[\mathcal{D} h_{\mu \nu}^{\perp}\right]$ measures are normalized by Gaussian conditions (3.12) in their respective spaces.

An interesting mathematical aspect of this construction of the covariant measure in quantum gravity is the natural appearance of the Yamabe condition (3.27) to fix completely the coordinates $g^{\perp}$ and $\sigma$ in the manifold of physical metric fields $\mathcal{M} / \mathcal{G}$. This problem of classical Riemannian geometry thus takes on an added importance at the quantum level. It would be very interesting to know, for example, if there are global obstructions to defining this coordinate system on the superspace $\mathcal{M} / \mathcal{G}$, since such obstructions would contain information 
about the global topology of the superspace which would have implications for the quantum theory in a non-perturbative domain. This is an area of mathematical investigation that has not been much explored, and which remains open for future research.

With the above methods of introducing a supermetric and volume element on the space of field configurations the path integral may be defined for systems with gauge and/or reparameterization invariance. It cannot be emphasized too strongly that in cases where the invariance group is non-compact, naive discretization methods fail completely and it is important to have some definition of the path integral where the integration is over gauge equivalence classes of configurations only. In the next section we shall consider the applications of this geometric method of defining the continuum path integral in both two and four dimensions.

\section{APPLICATIONS OF COVARIANT FUNCTIONAL INTEGRATION}

\section{A. The Conformal Anomaly in 2 Dimensions}

A simple application of the covariant path integral is the calculation of the trace anomaly in two dimensions, which leads to the Polyakov-Liouville action for quantum gravity in two dimensions. For this application we consider the invariant functional measure not over geometries but over matter fields. For example, consider a single free massless scalar field with the classical action,

$$
S_{c l}[g, \phi]=\int d^{2} x \sqrt{-g} g^{\mu \nu} \partial_{\mu} \phi \partial_{\nu} \phi
$$

which is clearly invariant under general coordinate transformations. In addition, it is also invariant under Weyl rescalings, since writing

$$
g_{a b}=e^{2 \sigma} \eta_{a b} ; \quad \sqrt{-g}=e^{2 \sigma}
$$

we observe that $S[\phi]$ is independent of $\sigma$. General coordinate invariance implies that the energy momentum tensor derived from $S[\phi]$ is covariantly conserved, 


$$
\nabla_{a} T^{a b}[g, \phi]=0 ; \quad T^{a b}[g, \phi]=\frac{2}{\sqrt{-g}} \frac{\delta S[g, \phi]}{\delta g_{a b}}=\partial_{a} \phi \partial_{b} \phi-\frac{1}{2}(\partial \phi)^{2} g_{a b}
$$

while Weyl invariance guarantees that this classical energy momentum tensor is traceless,

$$
g_{\mu \nu} T^{\mu \nu}[\phi]=e^{-2 \sigma} \frac{\delta S}{\delta \sigma}\left[g=e^{2 \sigma} \eta, \phi\right]=0 .
$$

Now we define the quantum effective action (in Euclidean time) by the covariant path integral,

$$
Z=\exp \left(-S_{e f f}[g]\right)=\int[\mathcal{D} \phi] \exp \left(-S_{c l}[g, \phi]\right)
$$

where the generally covariant integration measure over scalar fields must be defined such that

$$
\int[\mathcal{D} \phi] \exp \left(-\frac{1}{2}<\phi, \phi>\right)=1 ; \quad<\phi, \phi>\equiv \int d^{2} x \sqrt{+g} \phi^{2} .
$$

Now the point is that this inner product and the corresponding integration measure over scalar fields $[\mathcal{D} \phi]$ is invariant under general coordinate transformations but not under Weyl rescalings. Hence we must expect that the energy-momentum tensor of the quantized theory will remain conserved but have non-zero trace. Although this had to be discovered by laborious calculations in the standard operator quantization method, and for this reason was called the trace anomaly [17], it is actually obvious from the Weyl non-invariance of the covariant integration measure. Notice that it is logically possible to define a Weyl invariant scalar inner product and integration measure by leaving out the $\sqrt{+g}$ in (4.6) above, at the price of making it not generally coordinate invariant. In that case the quantum energy momentum tensor would remain traceless, but it would no longer be conserved. If coordinate invariance is assumed to be a more fundamental symmetry of nature than Weyl invariance this possiblity must be rejected.

In order to calculate the Weyl trace anomaly we perform the Gaussian integration in (4.5) and obtain

$$
S_{\text {eff }}[g]=\frac{1}{2} \operatorname{tr} \ln (-\square) \rightarrow-\frac{1}{2} \int_{\epsilon}^{\infty} \frac{d t}{t} \operatorname{tr} \exp (-t \square)
$$


where we have used the heat kernel definition of $\operatorname{tr} \ln$ and introduced a cutoff on the lower limit of integration. This regulated form is most convenient for evaluating the trace anomaly by varying with respect to $\sigma$. Using

$$
=\frac{1}{\sqrt{g}} \partial_{\mu}\left[\sqrt{g} g^{\mu \nu} \partial_{\nu}\right]=e^{-2 \sigma} \square
$$

with $\bar{\square}$ evaluated in the flat Euclidean metric, we find

$$
\begin{aligned}
g_{a b} T_{q u}^{a b} & =e^{-2 \sigma} \frac{\delta S_{\text {eff }}[g]}{\delta \sigma(x)} \\
& =-\int_{\epsilon}^{\infty} d t\left\langle x\left|\square e^{-t \square}\right| x\right\rangle \\
& =\left\langle x\left|e^{-\epsilon \square}\right| x\right\rangle \\
& =\frac{1}{4 \pi}\left[\frac{1}{\epsilon}+\frac{R}{6}+\mathcal{O}(\epsilon)\right],
\end{aligned}
$$

where the expansion of the heat kernel for $e^{-\epsilon \square}$ has been used in the last step as $\epsilon \rightarrow 0$, and we have assumed that the operator $\square$ has no zero modes so that the upper limit of the $t$ integral does not contribute. The background metric independent, divergent first term is again associated with the infinite energy density of the vacuum in flat space which can be regulated by the full $\zeta$-function method, or simply subtracted from the definition of the energy momentum tensor by a normal ordering procedure. The finite second term proportional to the Ricci scalar curvature of the background metric $g_{a b}$ is the trace anomaly. Notice that it indeed comes from the Weyl noninvariance of the integration measure (4.6),

since if we used the Weyl invariant measure omitting $\sqrt{g}$ from the inner product defined there, $\square$ in (4.8) above would be replaced by $\sqrt{g} \square=e^{2 \sigma} \square=\bar{\square}$ which is independent of $\sigma$, so that the variation in (4.9) would then give zero identically. The trace "anomaly" therefore is a necessary and immediate consequence of the covariant definition of the path integral measure in (4.6).

\section{B. The Path Integral for Quantum Gravity in Two Dimensions}

In the previous subsection the trace anomaly for one $\left(N_{S}=1\right)$ scalar field was calculated by the heat kernel method. The general form of the trace anomaly of the energy momentum 
tensor for classically Weyl invariant matter in a background gravitational field is

$$
\begin{aligned}
T_{a}^{a(\text { matter })} & =\frac{c_{m}}{24 \pi} R \\
& =\frac{c_{m}}{24 \pi} e^{-2 \sigma}(\bar{R}-2 \bar{\square} \sigma), \quad D=2
\end{aligned}
$$

in the decomposition $g_{a b}=e^{2 \sigma} \bar{g}_{a b}$. The coefficient $c_{m}=\left(N_{S}+N_{F}\right)$ for $N_{S}$ scalar and $N_{F}$ (Dirac) fermion fields. From eq. (4.9) this implies that there exists an effective anomalous quantum action such that

$$
\frac{\delta S_{\text {anom }}[g]}{\delta \sigma(x)}=\frac{c_{m}}{24 \pi}(\bar{R}-2 \bar{\square} \sigma)
$$

over and above any classical action for $\sigma$. Since the right side of this equation is linear in $\sigma$ we may integrate both sides immediately with respect to $\sigma$ to obtain the anomalous action:

$$
S_{\text {anom }}\left[g=e^{2 \sigma} \bar{g}\right]=S_{\text {anom }}[\bar{g}]+\frac{c_{m}}{24 \pi} \int d^{2} x \sqrt{-\bar{g}}[-\sigma \bar{\square} \sigma+\bar{R} \sigma] .
$$

Since $S_{\text {anom }}[g]$ must be a scalar under general coordinate transformations and a functional of only the full $g_{a b}$, we may use this information to determine the $\sigma$ independent integration constant $S_{\text {anom }}[\bar{g}]$ and write down the fully covariant but non-local form of the anomalous action:

$$
S_{\text {anom }}=-\frac{c_{m}}{96 \pi} \int d^{2} x \sqrt{-g} \int d^{2} x^{\prime} \sqrt{-g^{\prime}} R(x) \square^{-1}\left(x, x^{\prime}\right) R\left(x^{\prime}\right)
$$

If one adds to this induced action the classical Einstein-Hilbert action in two dimensions,

$$
\begin{aligned}
S_{c l} & =\int d^{2} x \sqrt{-g}(\gamma R-2 \lambda) \\
& =4 \pi \gamma \chi-2 \lambda \int d^{2} x \sqrt{-\bar{g}} e^{2 \sigma},
\end{aligned}
$$

one obtains the Polyakov-Liouville action which describes the fluctuations of random geometries, i.e. quantum gravity in two spacetime dimensions. Notice that the Einstein term alone describes no dynamics, since the integral of the scalar curvature is just $\chi$, the Euler number, and a topological invariant in $D=2$. Hence its local variation vanishes and one would obtain no equation of motion at all for the metric at the classical level. However, 
the addition of the anomalous action $S_{\text {anom }}[g]$ changes things dramatically. In its local form (4.13) we observe that a kinetic term for the $\sigma$ part of the metric has been generated by the path integration over the matter fields, and the dynamics of the metric can now be non-trivial.

Eq. (3.28) defines the covariant path integral for quantum gravity in a general number of spacetime dimensions. Restricting to $D=2$ Euclidean dimensions we observe that if the matter fields are considered as coordinates of a target space with dimension $c_{m}$, then we may also view the same expression as the partition function for non-critical closed bosonic string theory with a fixed world sheet topology. Summing $Z$ over the number of handles $h$ yields the full generating function for closed string amplitudes. It is the starting point for all of the modern analysis of string amplitudes in terms of the geometry of Riemann surfaces. The importance of the covariant point of view in elucidating the properties of string theory in terms of Riemannian geometry has been emphasized in the literature [18]. In the literature orthogonal coordinates on the space of metrics is generally used which implies $F=L^{\dagger}$, although this is by no means necessary. In these coordinates eq. (3.28) becomes

$$
Z=\left.\int[\mathcal{D} \sigma]\left[\mathcal{D} g_{\mu \nu}^{\perp}\right]\left[\operatorname{det}_{V}\left(L \circ L^{\dagger}\right)\right]^{+\frac{1}{2}}\right|_{g=e^{2 \sigma} g^{\perp}} \exp \left(-S_{i n v}\left[g=e^{2 \sigma} g^{\perp}\right]\right)
$$

where for $S_{i n v}$ we now put $-S_{a n o m}-S_{c l}$ above, after continuation to Euclidean time.

In two dimensions the kernel of the operator $L^{\dagger}$ is finite dimensional, being the space of quadratic differntials which is the tangent space to the Teichmüller space $\mathcal{T}$. This is defined by

$$
\mathcal{T}=\mathcal{M}_{\text {const }} / \mathcal{G}_{0}
$$

where $\mathcal{G}_{0}$ denotes the space of diffeomorphisms continuously connected to the identity and

$$
\mathcal{M}_{\text {const }} \equiv\left\{g^{\perp} ; R\left[g^{\perp}\right] \equiv \bar{R}=\text { const }\right\}
$$

is the Yamabe slice corresponding to the metric $g$ obtained by solving the uniformization (Yamabe) problem in two dimensions, namely [15] 


$$
R\left[g=e^{2 \sigma} g^{\perp}\right]=e^{-2 \sigma}(\bar{R}-2 \bar{\square} \sigma) .
$$

Since this always has a solution in $D=2$, the $\sigma$ and $g^{\perp}$ with constant $\bar{R}$ corresponding to any metric $g$ can always be uniquely determined. In fact the constant $\bar{R}$ can always be chosen to be +1 for two dimensional surfaces with $h=0$ handles (the topology of $S^{2}$ ), 0 for surfaces with $h=1$ handles (the topology of the torus $T^{2}$ ), and -1 for surfaces of higher genus with $h \geq 2$. Then it is known that

$$
\operatorname{dim}\left(\operatorname{ker} L^{\dagger}\right)=\operatorname{dim} \mathcal{T}= \begin{cases}0, & h=0, \\ 2, & h=1, \\ 6 h-6, & h \geq 2 .\end{cases}
$$

The basis tensors of the finite dimensional Teichmüller space are called moduli deformations, and may be denoted by $f_{j}$. Hence we may expand

$$
\delta g_{\mu \nu}^{\perp}=\sum_{j} \delta m_{j}\left(f_{j}\right)_{\mu \nu}
$$

where $\delta m_{j}$ denotes the modular deformation of the $j^{\text {th }}$ modular parameter over which we should finally integrate. Changing integration variables from $\delta g_{\mu \nu}^{\perp}$ to $\delta m_{j}$ generates a finite dimensional determinant in the integration measure, namely

$$
\left[\mathcal{D} g_{\mu \nu}^{\perp}\right]=\left[\operatorname{det}\left\langle\phi_{i} \mid \phi_{j}\right\rangle_{T}\right]^{-\frac{1}{2}} \operatorname{det}\left\langle f_{i} \mid \phi_{j}\right\rangle_{T} \prod_{j} d m_{j}
$$

where the $\phi_{i}$ are an arbitrary basis for ker $L^{\dagger}$, and the inner product for symmetric tensors is defined by Eq. (3.2) for $D=2$. This is the analog of the Jacobian factors (2.42) or (3.24) arising from the previous changes of variables to an arbitrary basis in the quotient space $\mathcal{M} / \mathcal{G}$. As in those cases it is possible (though not necessary) to choose an orthogonal basis depending on the field point in $\mathcal{M}$ in such a way as to reduce the Jacobian to a single determinant. Here the choice $\phi_{i}=f_{i}$ accomplishes that. The advantage of keeping the Jacobian in the more general form (4.22) is that the basis $\phi_{i}$ may be chosen in such a way that $\left\langle f_{i} \mid \phi_{j}\right\rangle$ is independent of the field point $g_{\mu \nu}=e^{2 \sigma} \bar{g}_{\mu \nu}$ and may removed from the functional integral, so that all the field dependence in contained in the first determinant alone. 
If $h=0,1$ then there are also globally defined conformal Killing fields obeying $L \psi=0$, since

$$
\operatorname{dim}(\operatorname{ker} L)= \begin{cases}6, & h=0, \\ 2, & h=1, \\ 0, & h \geq 2 .\end{cases}
$$

in accordance with the Riemann-Roch index theorem,

$$
\operatorname{dim}(\operatorname{ker} L)-\operatorname{dim}\left(\operatorname{ker} L^{\dagger}\right)=3 \chi=6(1-h) .
$$

The existence of conformal Killing fields implies that the coordinate chart $\left(\sigma, \xi, g^{\perp}\right)$ on the space $\mathcal{M}$ is not completely well-defined, since the decomposition (3.14) can no longer be inverted as in (3.17). Because this case is of some interest let us consider it in more detail.

First we should distinguish conformal Killing vectors with zero divergence, namely the Killing symmetries $\left(\nabla \cdot K_{i}=0, \mathrm{KV}\right.$ 's), from those with non-zero divergence, which we term proper conformal Killing vectors $\left(\nabla \cdot \psi_{i} \neq 0\right.$, PCKV's). The latter generate diffeomorphisms which are equivalent to a shift in $\sigma$. Hence the existence of PCKV's means that $\sigma$ has components along the gauge fiber $\mathcal{G}$, and we have not fully realized our aim of separating the gauge invariant field coordinates in $\mathcal{M} / \mathcal{G}$ from those along the fiber $\mathcal{G}$, or in physicist's language, we have not completely fixed the gauge. This arbitariness may be remedied easily enough by requiring that $\sigma$ be orthogonal to $\nabla \cdot \psi_{i}$ for each PCKV. Then by a change of coordinates in this finite dimensional space spanned by the $\psi_{i}$,

$$
[\mathcal{D} \sigma]_{\psi}=\tilde{J}[\mathcal{D} \psi]
$$

we find 20

$$
\tilde{J}=\left[\operatorname{det}_{V}\left\langle\psi_{i} \mid \psi_{j}\right\rangle\right]^{+\frac{1}{2}}
$$

by a calculation exactly similar to the previous ones leading to (2.42) and (3.24). In this way we can extract the volume of the gauge fiber projected onto the PCKV's $[\mathcal{D} \psi]$ from the scalar part of the measure $[\mathcal{D} \sigma]$ in (4.16), and are left with integrations only over those $\sigma$ orthogonal to the fiber and the finite dimensional Jacobian $\tilde{J}$. 
The true Killing symmetries (unlike the PCKV's) generate no change in the metric whatsover, so they cannot be treated in the same manner. Indeed the existence of KV's implies not that there is some residual gauge freedom in our coordinates $g^{\perp}$ and $\sigma$, but that the dimension of the gauge fiber $\mathcal{G}$ has decreased by precisely the number of linearly independent solutions of Killing's equation,

$$
\nabla_{\mu} K_{\nu}+\nabla_{\nu} K_{\mu}=0
$$

This is clearly a property of the geometry about which we are expanding, and not the choice of coordinates of that expansion. So, the KV's really should be treated differently from the PCKV's. In the string theory literature, the practice has been to treat all the CKV's on an equal footing, formally dividing $Z_{2}$ by the infinite volume of the non-compact Lie group corresponding to the full set of conformal Killing vectors $(S O(3,1) \simeq S L(2, C)$ in the case of $S^{2}$ ), so that the vacuum amplitude actually vanishes identically. Then, one shifts attention to non-vacuum matrix elements of reparameterization invariant vertex operators which lead to a compensating infinite factor in the numerator and finite results. Although the results so obtained are evidently correct (since they agree with older non-covariant operator calculations), this procedure of dividing one infinity by another clearly is not completely satisfactory, and it also leaves unanswered the question of how to treat the true Killing symmetries correctly. Let us for the moment ignore this subtlety and revisit the issue in more detail in the next section on semi-classical methods.

In order to make use of the expression (4.16) with (4.22) the $\sigma$ dependence of all the factors must be determined. This can again be done by the heat kernel method, the details of which may be found in the literature 18 . The result in $D=2$ is:

$$
Z_{2}=\int \prod_{j} d m_{j} J_{2}[\mathcal{D} \sigma]_{\bar{g}} e^{-c S_{a n o m}-S_{c l}}
$$

where the total Jacobian is

$$
\left.J_{2}=\left[\operatorname{det}\left\langle\bar{\phi}_{i} \mid \bar{\phi}_{j}\right\rangle_{T}\right]^{-\frac{1}{2}} \operatorname{det}\left\langle\bar{f}_{i} \mid \bar{\phi}_{j}\right\rangle_{T}\left\langle\bar{\psi}_{i} \mid \bar{\psi}_{j}\right\rangle_{V}\right]^{\frac{1}{2}}\left[\operatorname{det}_{V}^{\prime}\left(\bar{L}^{\dagger} \circ \bar{L}\right)\right]^{+\frac{1}{2}}
$$

and 


$$
c=c_{m}-26+1=N_{S}+N_{F}-25 .
$$

The -26 comes from the heat kernel expansion of $L^{\dagger} L$ and the additional +1 arises from the Weyl noninvariance of the $\sigma$ field itself which contributes to the anomaly coefficient just like one additional scalar field. Thus, the net effect of the $\sigma$ dependence of the measure for matter plus metric in $D=2$ dimensions is that we should replace $c_{m}$ by $c$ above, or in other words that the gravitational contribution to the trace anomaly is of the same form as that of the matter and would induce the Polyakov-Liouville action even in the complete absence of matter fields. Again, this is a purely quantum effect, arising in the path integral from the Weyl noninvariance of the proper covariant integration measure.

\section{The Semi-classical Partition Function on de Sitter Space}

Let us begin this subsection with Lorentzian signature and the usual Einstein-Hilbert action of general relativity,

$$
S_{c l}=\frac{1}{16 \pi G} \int_{\mathcal{M}} d^{4} x \sqrt{-g}(R-2 \Lambda)
$$

augmented by a positive cosmological term. The Euler-Lagrange variational principle $\delta S_{c l}=$ 0 yields the equations of motion,

$$
\begin{aligned}
R_{a b}-\frac{1}{2} R g_{a b} & =-\Lambda g_{a b} \\
\text { or } \quad R_{a b} & =\Lambda g_{a b},
\end{aligned}
$$

which admits a maximally symmetric solution, de Sitter spacetime with $O(4,1)$ isometry group. This classical solution is of particular interest in early universe cosmology.

Consider now expanding the action $S_{c l}$ to second order in metric fluctuations around the maximally symmetric de Sitter solution, utilizing the decomposition (3.14) and (3.15). We find

$$
32 \pi G \delta^{2} S=-<h^{\perp}, \Delta_{2} h^{\perp}>_{T}-24<\sigma,\left(-\Delta_{0}+\frac{1}{3} R\right) \sigma>_{S},
$$


where $\Delta_{2}$ is the tensor Lichnerowicz Laplacian,

$$
\left(\Delta_{2} h\right)_{a b}=\Delta_{0} h_{a b}+2 R_{a}{ }^{c d} h_{c d}+\frac{1}{2} R h_{a b}
$$

and $\Delta_{0}$ is the ordinary scalar Laplacian, $\Delta_{0}=-\nabla^{2}$. As expected, because of the general coordinate invariance of the action, $\delta^{2} S$ does not depend on the deformations generated by the diffeomorphisms $\xi$. However, the change of variables leaves behind a relic, namely the Jacobian factor $J$ given by (3.24) which becomes

$$
J=\left[\operatorname{det}_{V}^{\prime}\left(\bar{L}^{\dagger} \circ \bar{L}\right)\right]^{\frac{1}{2}}
$$

if orthogonal coordinates with respect to the inner product (3.2) are used to define $h^{\perp}$ and the CKV zero modes are excluded from the $\operatorname{det}_{V}^{\prime}$. Making the further change of variables in the $D+1=5$ dimensional space of PCKV's as described by (4.25) and (4.26) of the last subsection we obtain the semiclassical Gaussian functional integral of small fluctuations in Einstein gravity around de Sitter spacetime:

$$
Z^{s c}[d e S]=e^{i S_{c l}} \int J \tilde{J}[\mathcal{D} \sigma]^{\prime} \int\left[\mathcal{D} h^{\perp}\right] \exp \left(\frac{i}{64 \pi G}\left[-<h, \Delta_{2} h>+24<\sigma,\left(\Delta_{0}-\frac{1}{3} R\right) \sigma>\right]\right) .
$$

Naively one would think that the neglect of the higher orders of fluctuations in the exponent is justified in the limit of very weak Newtonian coupling $G \Lambda \rightarrow 0$. We shall see in a moment that this expectation will turn out to be incorrect.

Let us evaluate the Jacobian $J$ a bit more explicitly. The operator $\Delta_{1} \equiv L^{\dagger} L$ maps vectors into vectors. The space of all vectors may be decomposed into transverse and longitudinal vectors according to

$$
\xi_{a}=\xi_{a}^{\perp}+\nabla_{a} \phi
$$

A simple exercise in commuting covariant derivatives leads to the results,

$$
\left(\Delta_{1} \xi^{\perp}\right)_{a}=2\left(\Delta_{0}-\frac{1}{4} R\right) \xi_{a}^{\perp}
$$


and

$$
\left(\Delta_{1} \nabla \phi\right)_{a}=3 \nabla_{a}\left(\Delta_{0}-\frac{1}{3} R\right) \phi
$$

in the maximally symmetric de Sitter background. This means that the Jacobian expressed as a vector determinant may be rewritten as a product of determinants over transverse vectors $\xi^{\perp}$ and scalars $\phi$ :

$$
J=\left[\operatorname{det}_{V}^{\prime} \bar{\Delta}_{1}\right]^{\frac{1}{2}}=\left[\operatorname{det}_{\perp V}^{\prime}\left(\Delta_{0}-\frac{1}{4} R\right)\right]^{\frac{1}{2}}\left[\operatorname{det}_{S}^{\prime \prime}\left(\Delta_{0}-\frac{1}{3} R\right)\right]^{\frac{1}{2}}
$$

where an irrelevant real multiplicative constant has been discarded. The notation det ${ }_{S}^{\prime \prime}$ has been used to remind us that the original determinant of $L^{\dagger} L$ contains no zero modes or negative modes, so such modes cannot be present in the scalar determinant on the right side of the relation either. Notice otherwise that the scalar operator appearing in this determinant, $\Delta_{0}-\frac{R}{3}$ is the same as that appearing in the quadratic action (4.33). Hence the Gaussian integration over $[\mathcal{D} \sigma]^{\prime}$ in $(4.36)$ would cancel completely against this part of the Jacobian in the covariant measure, except for two subtleties. First, the sign of the operator $\Delta_{0}-\frac{R}{3}$ in (4.33) appears to be different from that in the Jacobian, and second, the integration $[\mathcal{D} \sigma]^{\prime}$ excludes zero modes but includes possible negative modes of the differential operator not included in the Jacobian, so any cancellation will not be complete.

The meaning of the cancellation is of course the same as found in the one loop partition function of QED, namely the role of the Jacobian is to cancel unphysical modes in the covariant decomposition of small fluctuations. In QED these are longitudinal modes which are eliminated in a canonical framework by Gauss' Law constraint. In Einstein gravity, one would expect the scalar modes of $\sigma$ to be eliminated by the constraints of diffeomorphism invariance in a canonical space plus time splitting. In the covariant functional integral this elimination shows up in the cancellation of scalar determinant by the correct Jacobian in the measure.

The question of the sign of the operator $\Delta_{0}-\frac{1}{3} R$ in (4.33) is related to the "conformal factor problem" of Einstein gravity which has received some attention (and caused a fair 
amount of confusion) in the literature, particularly in the context of continuation to Euclidean signature metrics [19]. Then the "wrong" sign would mean the Gaussian integral becomes exponentially divergent and consequently makes no sense at all. However, this is clearly an artificial problem and not a real difficulty since in the canonical approach to quantum gravity these scalar modes really are eliminated by the constraints (at least in perturbation theory around flat space) and cannot possibly lead to any physical instability in a correct covariant treatment. The resolution proposed [4] to this "conformal factor problem" is quite simple. In the definition of the inner product on the cotangent space of metric deformations (3.2) we required a metric (3.5) which contained an undetermined constant $C$. Since $C$ is not fixed by considerations of coordinate invariance alone, it can be determined only by additional information about the particular invariant action $S_{i n v}$ under consideration, i.e. it depends on dynamics and not pure kinematics, and may vary from theory to theory. In the Einstein theory the kinetic term for the $\sigma$ part of the action has the "wrong" sign, but canonical quantization methods precisely then imply that the constant $C=-\frac{2}{3}<-\frac{1}{2}$ in De Witt's supermetric (3.5) [16]. This means that the inner product

$$
<\delta g, \delta g>\left.\right|_{g}=<h^{\perp}, h^{\perp}>+<\xi, L^{\dagger} L \xi>+\frac{(1+2 C)}{4}<h, h>
$$

has a negative sign in its last term (in the scalar subsector of trace modes). Hence the conformal factor scalar Gaussian integration

$$
\int[\mathcal{D} \sigma] \exp \left(-\frac{i}{2} \frac{(1+2 C)}{4}<\sigma, \sigma>\right)=1
$$

is normalized with the opposite sign from the tensor modes

$$
\int\left[\mathcal{D} h^{\perp}\right] \exp \left(-\frac{i}{2}<h^{\perp}, h^{\perp}>\right)=1
$$

or in other words, the Euclidean continuation of the scalar modes should be performed in the opposite sense from the tensor modes in the semi-classical Einstein theory. This is the formal justification for the otherwise quite ad hoc prescription proposed by Hawking to integrate over imaginary conformal deformations [19]. The covariant functional integral makes it clear 
that some choice of $C$ is necessary and why the naive continuation to Euclidean signature is incorrect if $C<-\frac{1}{2}$. A fully consistent derivation of this condition from the BFV path integral in phase space has yet to be given however.

With the value of $C<-\frac{1}{2}$ the Euclidean continuation of the Gaussian integrations in (4.36) is unambiguous and well-defined, and the scalar determinants in (4.33) and $J$ do indeed cancel except for the subtlety of zero and negative modes. The Euclidean continuation of de Sitter spacetime is just $S^{4}$ with radius $a$ equal to $\sqrt{\frac{12}{R}}$. Hence the scalar operator $\Delta_{0}-\frac{R}{3}$ has the discrete spectrum, $[n(n+3)-4] / a^{2}$ with degeneracy $(2 n+3)(n+1)(n+$ $2) / 6$. The five zero modes at $n=1$ correspond precisely to the five PCKV's, $\nabla \cdot \psi_{i} \neq$ $0, i=1, \ldots 5$ which have been taken into account by the additional finite dimensional Jacobian $\tilde{J}$ [20]. The contribution of all of the positive eigenvalue modes at $n=2,3, \ldots$ are cancelled precisely by the same modes in the Jacobian $J$. We are then left with only the one negative eigenmode at $n=0$, corresponding to homogeneous expanding or shrinking of the radius of $S^{4}$. This mode is not eliminated by anything, and yields a divergent Gaussian integration corresponding to a genuine instability of the de Sitter background with respect to fluctuations in the average scalar curvature. Notice that this instability is infrared in character, having only to do with large scale fluctuations of the metric, and nothing at all to do with ultrashort Planck scale physics. For this reason, the results of the semiclassical analysis can be trusted, notwithstanding the ultraviolet nonrenormalizability of Einstein gravity. This infrared instability has been expected on other grounds [21], and is nicely confirmed in the covariant functional integral approach.

Ignored in this discussion of (4.36) is the question raised in the last subsection (in $D=2$ ) of how to treat the Killing vectors of de Sitter spacetime, or its Euclidean continuation to $S^{4}$. Non-trivial solutions to Killing's equation imply that a subgroup of the gauge group of diffeomorphism invariance $\mathcal{G}$ acts trivially at the field point possessing those Killing symmetries. Geometrically this is easy to visualize if we think of a simple analogy with a finite dimensional system possessing spherical symmetry. The symmetry group is $O(N)$ in $N$ dimensions and generates a gauge orbit with non-zero volume at every point of configuration 
space, except the origin. Near the origin the spherically symmetric invariant volume element goes to zero as $r^{N-1} d r$. At the origin it is identically zero because the invariance group acts trivially there. In the invariant path integral for quantum gravity, the factorization of the gauge orbit volume from the measure around a generic metric produces the Jacobian, $\operatorname{det}\left(L^{\dagger} L\right)$ which goes to zero when there are Killing symmetries. Hence, properly speaking, the invariant path integral measure around a metric with Killing symmetries actually vanishes identically. This means that a naive semiclassical evaluation of the functional integral neglecting the zero in the measure at the symmetric point cannot be correct.

To show this let us pursue a bit further the simple analogy with rotional invariance in a finite dimensional integral. Consider evaluating

$$
I(\epsilon)=2 \int_{0}^{\infty} d r r^{N-1} \exp \left(-\frac{r^{2}}{\epsilon}\right)
$$

where $\epsilon$ is assumed to be a small parameter. A naive steepest descent evaluation of this integral would consider only the saddle point at $r=0$, ignore the Jacobian measure factor and estimate $I \simeq \epsilon^{\frac{1}{2}}$. This is obviously incorrect since the integral is easily evaluated exactly:

$$
I(\epsilon)=\epsilon^{\frac{N}{2}} \Gamma\left(\frac{N}{2}\right)
$$

A much more accurate saddle point estimate for the integral is obtained by promoting the measure factor into the exponent and treating $1 / N$ and $\epsilon$ as small parameters of the same order. Then the modified saddle point occurs at non-zero $r_{0}^{2}=(N-1) \epsilon / 2$ and we obtain the estimate,

$$
I \approx 2 \epsilon^{\frac{N}{2}}\left(\frac{\pi}{2}\right)^{\frac{1}{2}}\left(\frac{N-1}{2}\right)^{\frac{N-1}{2}} e^{-\frac{N-1}{2}}
$$

which is clearly more accurate than the naive estimate, gives the correct $\epsilon$ dependence for any $N$, and a numerical prefactor which agrees with the exact answer in the limit $N \rightarrow \infty$. Even for $N=2$ the saddle point estimate gives the numerical prefactor $\sqrt{\frac{\pi}{e}}=1.075$ compared to the exact $\Gamma(1)=1$.

The important lesson of this simple example is that it is not correct to do the naive saddle point expansion around a point where the measure vanishes, since the measure shifts 
the correct saddle point away from the most symmetric point. Expanding the invariant path integral for quantum gravity around a geometry with normalizable Killing vectors and ignoring the zero in the Jacobian measure is just such a mistake, since the quantum measure vanishes there and must be taken into account to find the correct non-symmetric saddle point. The de Sitter geometry gives precisely zero contribution to the partition function for quantum gravity because it has zero weight in the functional integral, when the correct invariant measure is taken into account. This phenomenon is related to the linearization instability of the classical solution in the canonical approach to classical gravity [22], whose interpretation in the full quantum theory has remained somewhat obscure. Notice that there is no such problem in flat space since the Killing vectors in that case are not normalizable with respect to the inner product (3.22) which defines the integration measure on the fiber of gauge equivalence $\mathcal{G}$. Hence there is no linearization or other instability of flat spacetime [3.

Finally, as we have seen, the $\sigma$ zero mode did not cancel against anything and has a Gaussian with the sign corresponding to instability of the de Sitter background. Hence the quantum state of the gravitational field of linearized fluctuations around de Sitter space cannot be de Sitter invariant. The consequences of this infrared behavior in de Sitter space for quantum gravity in the large is the subject of the next section. Once again we see that the invariant functional integration measure has physical consequences which may be exposed directly, without the cumbersome calculations of noncovariant approaches.

\section{The Effective Theory of the Conformal Factor in Four Dimensions}

We have seen that the diffeomorphism constraints encoded in the Jacobian of change of variables $J$ does not completely cancel the excitations of $\sigma$ around de Sitter space, so that the quantum theory does not preserve exactly the same diffeomorphism constraints as the classical Einstein theory. The surviving mode is an infrared mode corresponding to dilation of the entire space. The existence of an infrared instability in the quantum 
fluctuations around de Sitter spacetime indicates that there is nontrivial dynamics in the conformal sector of four dimensional gravity. In other words, there is an infrared anomaly in the trace sector, and we would expect the effects of this anomaly to be most important at the largest distance scales. The consequences of this anomaly for the vacuum energy or cosmological constant problem may be addressed most directly in the invariant functional integral approach as well.

The line of reasoning followed in $D=2$ to find the conformal anomaly and construct the Polyakov-Liouville effective action of two dimensional quantum gravity may be followed also for $D=4$. First the general form of the trace anomaly of the energy-momentum tensor in four dimensions may be calcaluted by heat kernel regularization of the determinants found by covariant functional integration over matter fields of arbitrary spin propagating on a fixed curved manifold. A linear combination of the four invariants, $R^{2}, R_{a b} R^{a b}, R_{a b c d} R^{a b c d}$, and $\square R$ is obtained $\llbracket 17$. Actually, since $\square R$ is a total divergence in any number of dimensions, adding it to the effective action gives no contribution to the trace of the energy momentum tensor. Hence there can be at most three independent local terms in the general trace anomaly, which therefore may be written in the form [23],

$$
T_{a}^{a(\text { matter })}=b\left(F+\frac{2}{3} \square R\right)+b^{\prime} G+b^{\prime \prime} \square R, \quad D=4
$$

where

$$
F=R_{a b c d} R^{a b c d}-2 R_{a b} R^{a b}+\frac{1}{3} R^{2}
$$

is the square of the Weyl tensor in four dimensions, and

$$
G=R_{a b c d} R^{a b c d}-4 R_{a b} R^{a b}+R^{2}
$$

is the Gauss-Bonnet integrand. In four dimensions $G$ is a total divergence, and its integral is a topological invariant, proportional to the Euler number. Hence we expect its coefficient $b$ to play a role similar to the $c$ in two dimensions. The coefficients $b$ and $b^{\prime}$ have been computed for scalar, Dirac fermion, and vector fields at one-loop order, with the results [23]: 


$$
\begin{aligned}
b & =\frac{1}{120(4 \pi)^{2}}\left(N_{S}+6 N_{F}+12 N_{V}\right), \\
b^{\prime} & =-\frac{1}{360(4 \pi)^{2}}\left(N_{S}+11 N_{F}+62 N_{V}\right) .
\end{aligned}
$$

If the matter field Lagrangian is invariant under local conformal (Weyl) transformations classically, then the coefficient $b^{\prime \prime}$ vanishes at one-loop order. However, in principle it corresponds to an arbitrary parameter, since $\square R$ is the variation of a local action:

$$
\sqrt{-g} \square R=-\frac{1}{6} g_{a b} \frac{\delta}{\delta g_{a b}} \int d^{4} x \sqrt{-g} R^{2},
$$

which receives divergent contributions in general. Thus we allow for $b^{\prime \prime} \neq 0$. In contrast to this term, the first two terms on the right side of (4.47) do not arise from local effective actions in four dimensions. However, in the conformal parameterization of the metric, the anomaly may be derived from a local effective action, just as in the two dimensional case [24.25]. In fact, if we rewrite the trace anomaly (4.47) in the form,

$$
\begin{aligned}
T_{a}^{a(\text { matter })} & =b F+b^{\prime}\left(G-\frac{2}{3} \square R\right)+\left[b^{\prime \prime}+\frac{2}{3}\left(b+b^{\prime}\right)\right] \square R \\
& =\frac{1}{\sqrt{-g}} \frac{\delta}{\delta \sigma(x)} S_{\text {anom }}[\sigma],
\end{aligned}
$$

the first term is independent of $\sigma$, the second term is linear in $\sigma$, and are easily integrated, while the $\sigma$ dependence of the third term is determined by eq. (4.51). Hence the full anomaly induced action in four dimensions reads:

$$
\begin{aligned}
& S_{\text {anom }}[\bar{g}, \sigma]=S_{\text {anom }}[\bar{g}] b \int d^{4} x \sqrt{-\bar{g}} \bar{F} \sigma \\
& +b^{\prime} \int d^{4} x \sqrt{-\bar{g}}\left\{\sigma\left[2 \bar{\square}^{2}+4 \bar{R}^{a b} \bar{\nabla}_{a} \bar{\nabla}_{b}-\frac{4}{3} \bar{R} \bar{\square}^{2}+\frac{2}{3}\left(\bar{\nabla}^{a} \bar{R}\right) \bar{\nabla}_{a}\right] \sigma+\left[\bar{G}-\frac{2}{3} \bar{\square} \bar{R}\right] \sigma\right\} \\
& \quad-\frac{1}{12}\left[b^{\prime \prime}+\frac{2}{3}\left(b+b^{\prime}\right)\right] \int d^{4} x \sqrt{-\bar{g}}\left[\bar{R}-6 \bar{\square} \sigma-6\left(\bar{\nabla}_{a} \sigma\right)\left(\bar{\nabla}^{a} \sigma\right)\right]^{2},
\end{aligned}
$$

where we have used the expression,

$$
R=e^{-2 \sigma}\left(\bar{R}-6 \bar{\square} \sigma-6\left(\bar{\nabla}_{a} \sigma\right)\left(\bar{\nabla}^{a} \sigma\right)\right),
$$

for the Ricci scalar in conformal coordinates. We can again use the fact that the action must be an invariant function of the total metric $\bar{g} e^{2 \sigma}$ to write down the fully covariant anomaly-induced non-local action, 


$$
\begin{aligned}
S_{\text {anom }}= & -\frac{1}{4} \int d^{4} x \sqrt{-g} \int d^{4} x^{\prime} \sqrt{-g^{\prime}}\left[b F+b^{\prime}\left(G-\frac{2}{3} \square R\right)\right]_{x} \\
& {\left[2 \square^{2}+4 R^{a b} \nabla_{a} \nabla_{b}-\frac{4}{3} R \square^{2}+\frac{2}{3}\left(\nabla^{a} R\right) \nabla_{a}\right]_{x x^{\prime}}^{-1}\left[b F+b^{\prime}\left(G-\frac{2}{3} \square R\right)\right]_{x^{\prime}} } \\
& -\frac{1}{12}\left[b^{\prime \prime}+\frac{2}{3}\left(b+b^{\prime}\right)\right] \int d^{4} x \sqrt{-g} R^{2} .
\end{aligned}
$$

The non-local term in (4.55) is the four dimensional analog of the non-local form of the $D=2$ Polyakov action, (4.14). As in two dimensions we must add the anomaly induced action (4.53) to the classical Einstein-Hilbert action,

$$
\begin{aligned}
S_{c l} & =\frac{1}{2 \kappa} \int d^{4} x \sqrt{-g}(R-2 \Lambda) \\
& =\frac{1}{2 \kappa} \int d^{4} x \sqrt{-\bar{g}} e^{2 \sigma}\left(\bar{R}-6 \bar{\square} \sigma-6\left(\bar{\nabla}_{a} \sigma\right)\left(\bar{\nabla}^{a} \sigma\right)\right)-\frac{\Lambda}{\kappa} \int d^{4} x \sqrt{-\bar{g}} e^{4 \sigma},
\end{aligned}
$$

where $\kappa=8 \pi G$.

In the case that the fiducial metric is conformally flat, i.e. $\bar{g}_{a b}=e^{2 \bar{\sigma}} \eta_{a b}$, the effective action for $\sigma$ may be obtained by a translation, $\sigma \rightarrow \sigma+\bar{\sigma}$ of the following simpler flat space action,

$$
\begin{aligned}
S_{e f f} & =S_{\text {anom }}+S_{c l} \\
& =\int d^{4} x\left\{2 b^{\prime}(\square \sigma)^{2}-\left[3 b^{\prime \prime}+2\left(b+b^{\prime}\right)\right]\left(\square \sigma+\left(\partial_{a} \sigma\right)^{2}\right)^{2}+\frac{3}{\kappa} e^{2 \sigma}\left(\partial_{a} \sigma\right)^{2}-\frac{\Lambda}{\kappa} e^{4 \sigma}\right\},
\end{aligned}
$$

up to a surface term.

The first important property of this effective action we should note is that it is completely bounded, unlike the classical Einstein-Hilbert action when continued naively to Euclidean signature metrics.

There is no conformal factor problem in the effective theory of the conformal factor generated by a proper treatment of the measure of the covariant path integral and trace anomaly in four dimensional gravity.

In the language of the previous section the constant $C$ of Eq. (3.5) should be chosen greater than one half,unlike in the Einstein theory, in the full effective field theory of the conformal factor generated by $S_{a n o m}$ in four dimensions, so that the normal continuation to Euclidean signature applies. Again, the proper derivation of this choice requires a careful 
consideration of the canonical formulation of the path integral in phase space which has not yet been done and remains an open problem. This justification is important for comparison to the necessarily Euclidean dynamical triangulation approach as well.

Notice also from the derivation of $S_{\text {anom }}$ that although the $\sigma$ independent piece of the gravitational action cannot be determined from the trace anomaly alone, the $\sigma$ dependence is uniquely determined by the general form of the trace anomaly for massless fields. Thus, whatever else may be involved in the full quantum theory of gravity in four dimensions at short distance scales, the anomalous effective action (4.53) must be included in the gravitational action at large distance scales, i.e. in the far infrared, since the gravitational effects of massless fields do not decouple and are screened by nothing at large distances. Graviton (i.e. spin-two) fluctuations of the metric should give rise to an effective action of precisely the same form as $S_{\text {anom }}$ with new coefficients $b$ and $b^{\prime}$, which can be checked at one-loop order [8]. The effective action (4.55) or (4.57) following from the invariant path integral for quantum gravity in the conformal sector is the starting point for finite volume scaling and infrared renormalization group comparisons to the dynamical triangulation approach.

\section{E. Finite Volume Scaling and Infrared Renormalization}

An interesting property of the expression (4.28) for the functional integral over geometries of fixed topology in two dimensions is that the total trace anomaly of matter plus ghosts plus $\sigma$ vanishes identically. To see this we have only to realize that the effective action with arbitary coefficient,

$$
c S_{\text {anom }, E u c l}=+\frac{Q^{2}}{4 \pi} \int d^{2} x \sqrt{\bar{g}}\left[(\bar{\nabla} \sigma)^{2}+\bar{R} \sigma\right]
$$

has an trace anomaly coefficient,

$$
c_{\sigma}=1+6 Q^{2}
$$

where the 1 is the quantum contribution of a single scalar field coming from the kinetic

term as computed in the last section by the heat kernel method, and $+6 Q^{2}$ is the "classical" 
contribution which arises from the simple fact that $S_{\text {anom }}$ is not classically Weyl invariant because of the linear $\bar{R} \sigma$ term. If we now set the total anomaly equal to zero

$$
c_{m}+c_{g h}+c_{\sigma}=\left(N_{S}+N_{F}\right)+(-26)+\left(1+6 Q^{2}\right)=0
$$

we obtain precisely the value of $Q^{2}=-c / 6$ found in (4.30). The vanishing of the total trace anomaly coefficient is very important, since it implies that conformal invariance is restored in the full quantum theory, when the metric field $e^{2 \sigma} \bar{g}$ is quantized by the invariant functional integral method. If conformal invariance is restored then the quantum theory describes a fixed point of the renormalization group flow, about which we shall say more in the next section. Another consequence is that all the allowed operators in the theory must have well-defined conformal dimension or weight. The conformal weight of an operator $X$ is defined by the behavior of $X$ under a coordinate transformation,

$$
\delta_{\xi} X=\xi^{a} \nabla_{a} X+\left(\nabla_{a} \xi^{a}\right) \frac{w(X)}{D} X
$$

In classical relativity this just defines the transformation property of a scalar density of weight $w(X)$. Thus $e^{D \sigma}=\sqrt{g}$ transforms as a density of weight $D$ in $D$ dimensions, classically.

In the quantum theory this same transformation is a canonical transformation generated by the corresponding moment of the energy momentum tensor $T^{a b}$, viz.

$$
\delta_{\xi} X=i\left[T_{\xi}, X\right], \quad \text { with } \quad T_{\xi}=\int_{\Sigma} d \Sigma_{b} \xi_{a} T^{a b}
$$

where $\Sigma$ is an arbitrary spacelike Cauchy surface in the space plus time foliation of the geometry. Because the anomalous action and its corresponding energy-momentum tensor differ from the classical ones, the conformal weight $w(X)$ computed this way in the quantum theory will differ in general from the classical weight. In fact, a simple computation shows that for a pure exponential operator

$$
w\left(e^{p \sigma}\right)=p-\frac{p^{2}}{2 Q^{2}},
$$


in place of the classical result which is just $p$. However, only those operators with conformal weight equal to 2 in $D=2$ dimensions can be integrated with respect to $d^{2} x$ to yield a coordinate invariant scalar, which may be added to the invariant action. This implies that the cosmological term in the Polyakov-Liouville action must be modified to

$$
\int d^{2} x e^{2 \alpha \sigma} \sqrt{\bar{g}}
$$

where $\alpha$ is determined by requiring the conformal weight to be 2 :

$$
w\left(e^{2 \alpha \sigma}\right)=2 \alpha-\frac{2 \alpha^{2}}{Q^{2}}=2,
$$

or

$$
\alpha_{ \pm}=\frac{1 \pm \sqrt{1-\frac{4}{Q^{2}}}}{\frac{2}{Q^{2}}}
$$

The negative branch of the square root goes over to the classical result,

$$
\alpha_{-} \rightarrow 1 \quad \text { as } \quad Q^{2} \rightarrow \infty
$$

As one simple example of the consequences of this anomalous dimension we may restrict ourselves to the simplest topology, $S^{2}$ with $h=0$, and consider the fixed area partition function with $S_{c l}=0$ as well,

$$
Z_{2}(A) \equiv \int J_{2}[\mathcal{D} \sigma]_{g^{\perp}} \exp \left(-c S_{\text {anom }}\right) \delta\left(\int d^{2} x \sqrt{-\bar{g}} e^{2 \alpha \sigma}-A\right)
$$

By a simple constant shift in $\sigma$,

$$
\sigma \rightarrow \sigma+\frac{\omega}{\alpha}
$$

we find that $Z(A)$ obeys the scaling relation,

$$
Z(A)=e^{-2 \omega\left[Q^{2}(1-h) / \alpha+1\right]} Z\left(e^{-2 \omega} A\right)
$$

which implies

$$
Z(A) \propto A^{-\left[Q^{2}(1-h) / \alpha+1\right]} \equiv A^{\Gamma(h)-3}
$$


where $\Gamma(h)$ is conventionally called the string susceptibility for surfaces with genus $h$. For surfaces of genus 0 , i.e. the topology of $S^{2}$, we obtain

$$
\Gamma(0)=-Q^{2} / \alpha+2=\left[c_{m}-1-\sqrt{\left(25-c_{m}\right)\left(1-c_{m}\right)}\right] / 12,
$$

if the negative sign of the square root is chosen for $\alpha$. This scaling had been obtained earlier by operator methods in a much more difficult calculation [27]. By using the properties of the covariant path integral for $D=2$ dimensional quantum gravity the same result is almost immediate [26].

Let us now study the consequences of the quantization of the conformal factor with the four dimensional action (4.57). The trace of the energy momentum tensor of this effective scalar theory must vanish, because of the coordinate invariance of the full theory, for the same reason as in the $D=2$ case, and as may be explicitly verified in $D=4$ again. This implies that the beta functions of all couplings must vanish, but does not preclude the possibility of nontrivial anomalous scaling dimensions. The anomalous scaling dimension $\alpha$ in $D=4$ dimensions may be determined by a calculation only slightly more complicated than the corresponding one in 2 dimensions, by requiring that the operator, $\sqrt{-g} R=$ $-6 e^{2 \alpha \sigma}\left((\partial \sigma)^{2}+\square \sigma\right)$ have conformal weight equal to four. Indeed, direct calculation of this conformal weight shows that the condition is [25]

$$
w(\sqrt{-g} R)=2 \alpha-\frac{2 \alpha^{2}}{Q^{2}}+2=4 .
$$

This is the necessary condition for the Einstein term to be invariant under the conformal diffeomorphism symmetry (4.62). Equivalently, one may calculate the logarithmic divergences contributing to the renormalization of the $\gamma \equiv \frac{3}{\kappa}$ coupling multiplying the same Einstein term in the classical action and obtain the following beta function condition:

$$
\beta_{\gamma}=\mu \frac{d}{d \mu} \gamma_{R}=\left(2-2 \alpha+\frac{2 \alpha^{2}}{Q^{2}}\right) \gamma_{R}=0 .
$$

The first two terms correspond to the "classical" scaling dimenension of the $\gamma$ coupling, for $\alpha \neq 1$, while the $\alpha^{2}$ term arises from the one-loop counterterm. The vanishing of this beta function for $\gamma_{R} \neq 0$ yields a quadratic relation for the anomalous scaling of $e^{\sigma}$ [25]: 


$$
1-\alpha+\frac{\alpha^{2}}{Q^{2}}=0,
$$

which is precisely the same quadratic equation for $\alpha$ as in 2 dimensions, provided $Q^{2}$ is defined in $D=4$ by the anomaly coefficient: of the Gauss-Bonnet term in (4.47).

$$
Q^{2}=-32 \pi^{2} b^{\prime}
$$

One observation we can make at this point is that if the expectation value of the Ricci scalar is different from zero, then the conformal symmetry must be spontaneously broken. In the semiclassical limit, when the anomaly induced fluctuations are suppressed by $\frac{1}{Q^{2}}$ for large $Q^{2}, \alpha \rightarrow 1$ and the weight of $R$ is zero, i.e. it transforms like a scalar under global conformal transformations. If $\alpha$ differs from unity, then this is no longer the case. This implies that $\langle R\rangle$ becomes an order parameter for the spontaneous breaking of global conformal symmetry, in sharp contrast to the classical situation in which $\langle R\rangle$ can take on any value consistent with the symmetry. In the classical Einstein equations $R$ is just proportional to the cosmological constant. Because of the trace anomaly induced by the coordinate invariant (but Weyl-noninvariant) measure, the cosmological "constant" problem reduces to the question of whether conformal symmetry is spontaneously broken, or restored in the ground state of quantum gravity.

Consider now the correlation function of Ricci scalars, $\left\langle R(x) R\left(x^{\prime}\right)\right\rangle$ at two different points. Using the expression, (4.54) with $\sigma$ replaced by $\alpha \sigma$, and the free action which describes the theory at its critical point, we find:

$$
\begin{aligned}
\left\langle R(x) R\left(x^{\prime}\right)\right\rangle & \rightarrow \bar{R}^{2}\left\langle e^{-2 \alpha \sigma(x)} e^{-2 \alpha \sigma\left(x^{\prime}\right)}\right\rangle \\
& \rightarrow \bar{R}^{2} e^{4 \alpha^{2}\left\langle\sigma(x) \sigma\left(x^{\prime}\right)\right\rangle} \\
& =\bar{R}^{2}\left|\frac{H s\left(x, x^{\prime}\right)}{2}\right|^{-\frac{4 \alpha^{2}}{Q^{2}}}, \quad\left|s\left(x, x^{\prime}\right)\right| \rightarrow \infty,
\end{aligned}
$$

where only the dominant infrared behavior has been retained, and $s\left(x, x^{\prime}\right)$ is the invariant distance between the points $x$ and $x^{\prime}$.

The result (4.77) states that the effective cosmological "constant" goes to zero with a definite power law behavior for large distances. In other words, there is screening in the infrared 
of the effective value of vacuum energy at larger and larger scales. The value of the power is universal, depending only on $Q^{2}$ which counts the effective number of massless degrees of freedom. In particular, it depends neither on the classical value of the background curvature $\bar{R}$, nor on the Planck scale. This is essential for a scale invariant, phenemenologically acceptable solution of the cosmological constant problem.

These results were obtained in the continuum by treating the metric $\bar{g}_{a b}$ as fixed. In other words the transverse, traceless sector of the theory containing the physical spin-2 gravitons was neglected completely. Our basic hypothesis is that these relations remain true in the infrared when the graviton modes are included, up to a possible renormalization of the value of $Q^{2}$. More precisely we assume that integration over the transverse graviton modes generates an effective action for $\sigma$ which, when expanded in powers of derivatives, has the same form as (4.53) but with renormalized coefficients. This assumption we have called "infrared conformal dominance [8]."

Such an assumption is not at all unreasonable from a Wilsonian effective action point of view. Consider the functional integration over transverse gravitons (in other words over conformal equivalence classes of metrics), as well as matter fields, with both infrared and ultraviolet cut-offs, $\ell$ and $a$. At short distances, graviton effects may grow uncontrollably due to the presence of the dimensionful Newtonian coupling $\kappa$, so that a cannot be taken to zero in the effective action. Conversely, at large distance scales, the transverse, tracefree fluctuations should be expected to become less important, so that the effective action should be regular as the infrared cutoff $\ell$ is removed. If this is the case, then an infrared stable renormalization group fixed point of the effective low energy theory is approached as $\ell \rightarrow \infty$. Scale invariance at this fixed point then requires that the low energy effective action must be of the form (4.53) when expanded up to four derivatives of $\sigma$. Yet, infrared conformal dominance is still at this point an assumption which can be checked in principle, by finding the scaling behavior of the invariant functional integral for quantum gravity in the large $\ell$ or infinite volume limit, as the infrared fixed point is approached.

In order to derive the scaling behavior of the partition function of the effective $\sigma$ theory 
in $D=4$, we require one more anomalous dimension because of the additional term in the classical action in $D=4$ as compared with $D=2$. This is the anomalous dimension of the volume operator in four dimensions, which we denote by $\beta$ (not to be confused with the $\beta$ function defined previously). The weight of the pure exponential volume operator is determined by using Eq. (4.63) which gives

$$
w\left(\sqrt{\bar{g}} e^{4 \beta \sigma}\right)=4 \beta-\frac{(4 \beta)^{2}}{2 Q^{2}}=4,
$$

since the normalization of $Q^{2}$ has been adjusted to give the same relations in $D=4$ as $D=2$. Hence we find that in order to add the volume or cosmological term to the effective action, it must have the anomalous dimension [28]

$$
\beta_{ \pm}=\frac{1 \pm \sqrt{1-\frac{8}{Q^{2}}}}{\frac{4}{Q^{2}}}
$$

where we must again choose the negative sign of the square root in order to recover the classical result,

$$
\beta_{-} \rightarrow 1 \quad \text { as } \quad Q^{2} \rightarrow \infty
$$

Armed with this information we may now subject the $\sigma$ field to the analog of the constant shift (4.69) performed in two dimensions,

$$
\sigma \rightarrow \sigma+\frac{\omega}{\beta}
$$

and use the translational invariance of the invariant integration measure $[\mathcal{D} \sigma]$ to find

$$
Z(\kappa, \lambda) \equiv \int[\mathcal{D} \sigma] e^{-S_{e f f}[\sigma]}=e^{-\frac{Q^{2}}{\beta} \chi \omega} Z\left(\kappa e^{-2 \frac{\alpha \omega}{\beta}}, \lambda e^{4 \omega}\right)
$$

If we restrict to the fixed topology of $S^{4}$ for which $\chi=2$, and define also the partition function at fixed volume

$$
Z(\kappa ; V) \equiv \int[\mathcal{D} \sigma] e^{\lambda V-S_{e f f}[\sigma]} \delta\left(\int d^{4} x \sqrt{\bar{g}} e^{4 \beta \sigma}-V\right)
$$

we obtain 


$$
\begin{aligned}
Z(\kappa ; V) & =e^{-2 \omega\left(\frac{Q^{2}}{\beta}+2\right)} Z\left(\kappa e^{-2 \frac{\alpha \omega}{\beta}} ; e^{-4 \omega} V\right) \\
& =V^{-\left(\frac{Q^{2}}{2 \beta}+1\right)} \tilde{Z}\left(\kappa V^{-\frac{\alpha}{2 \beta}}\right) \\
& =V^{-\left(4+Q^{2}+Q \sqrt{Q^{2}-8}\right) / 4} \tilde{Z}\left(\kappa V^{-\frac{\alpha}{2 \beta}}\right)
\end{aligned}
$$

where in the second line we put $e^{4 \omega} \propto V$. This analog of the scaling relation (4.71) for $D=4$ should be testable by the dynamical triangulation approach to the functional integration over four geometries which is the subject of the last section. Notice also that the limit of very large volume $V \rightarrow \infty$ is equivalent to the limit of zero Newtonian constant $\kappa \rightarrow 0$. This is simply the statement that for scales $\ell>>L_{\text {Planck }} \simeq a$, the ultraviolet cutoff scale becomes irrelevant and scaling is determined purely by the infrared fixed point of the theory, the anomalous dimensions there and the corresponding value of $Q^{2}$, in complete accord with the behaviour of critical exponents in the Wilson theory of second order phase transitions [1]. The effective vanishing of the Newtonian coupling in the large volume limit implies that ordinary perturbation theory should be applicable to the calculation of the graviton contribution to the conformal anomaly in the far infrared [8]. The analytic calculation of this contribution and the complete determination of $Q^{2}$ and therefore the scaling in (4.84) remains an important unresolved issue, and of course one that should have consequences for large scale structure in the universe.

\section{THE DYNAMICAL TRIANGULATION DEFINITION OF THE FUNCTIONAL INTEGRAL}

A completely different approach to defining the path integral for reparameterization invariant theories such as quantum gravity is the dynamical triangulation approach. This is a discretization method that avoids the introduction of coordinates on manifolds, defining the functional integration directly over distinct geometries. This approach has the great advantage of avoiding the gauge fixing problem of factorizing out the infinite volume of the gauge fiber $\mathcal{G}$. The corresponding disadvantage is that there is no meaning to reparameter- 
ization invariance in the dynamical triangulation approach and the integration measure is not determined a priori, or even necessarily related to the continuum measure constructed in the previous sections.

Dynamical triangulation is a variation of Regge calculus in which geometries are constructed by gluing together fundamental simplices of fixed volume [5]. In two dimensions the regular simplices are just equilateral triangles. In four dimensions the four-simplices share common faces with their neighbors which are $D-1=3$ dimensional simplices, i.e. regular tetrahedra of fixed edge length $a$. The angle between two tetrahedra faces sharing a triangle is

$$
\theta=\arccos \left(\frac{1}{D}\right)=1.3181161
$$

in $D=4$ dimensions. The volume of a fundamental $J$-simplex is

$$
V_{J}=a^{J} \Omega_{J}=\frac{a^{J}}{J !} \sqrt{\frac{J+1}{2^{J}}},
$$

so that the total volume of the simplicial manifold is

$$
\int d^{4} x \sqrt{g} \rightarrow N_{4} V_{4}
$$

if $N_{4}$ is the total number of 4 -simplices in the configuration.

As in the Regge approach, space is regarded as flat inside the $D=4$ simplices with all the curvature residing on the $D-2=2$ dimensional hinges, i.e. equilateral triangles with the same fixed edge length. If $n_{i}$ is the number of 4-simplices sharing a given equilateral triangle $i$, then the deficit angle $\delta_{i}$ is given by

$$
\delta_{i}=2 \pi-n_{i} \theta
$$

and the Einstein-Hilbert action takes the value,

$$
\int d^{4} x \sqrt{g} R \rightarrow \sum_{i} \delta_{i} V_{2}=\left(2 \pi N_{2}-10 \theta N_{4}\right) V_{2}
$$

where $\sum_{i} n_{i}=10 N_{4}$ has been used (since each 4-simplex has 10 triangles in its boundary). 
Dynamics is now specified by giving an action for each simplicial triangulation $\mathcal{T}$ of the form $S(\mathcal{T})=\sum_{J} k_{J} N_{J}(\mathcal{T})$, and summing over all triangulations weighted by this action with each triangulation otherwise having equal statistical weight. In this approach there is no diffeomorphism invariance at the lattice level, and no gauge-fixing problems. As a corollary, neither is it self evident that the statistical model(s) constructed by the dynamical triangulation procedure lie in the same universality class as quantum gravity in four dimensions. Certainly a necessary condition for that to hold is that the simplicial complexes generated should approximate a continuous manifold as the lattice becomes finer and finer. This forces some of the numbers of $J$-subsimplices (for $J=0,1, \ldots, D$ ) to satisfy several relations, called Dehn-Sommerville relations, so that not all of the $N_{J}$ are independent [29]. In addition we have the Euler relation,

$$
\sum_{J=0}^{4}(-)^{J} N_{J}=\chi .
$$

The net result is that only two of the $N_{J}$ are independent and the action may be taken to be of the form,

$$
S(\mathcal{T})=-k_{2} N_{2}(\mathcal{T})+k_{4} N_{4}(\mathcal{T}) .
$$

Comparing this with the Einstein-Hilbert action, and using the substitutions (5.3) and (5.5) leads to the following identification of the bare simplicial action parameters with the (unrenormalized) parameters of the continuum theory,

$$
\begin{aligned}
& k_{2}=\frac{\sqrt{3} \pi}{4} \frac{a^{2}}{\kappa} \\
& k_{4}=\frac{5 \sqrt{3}}{4} \theta \frac{a^{2}}{\kappa}+\frac{\sqrt{5}}{96} \lambda a^{4}
\end{aligned}
$$

where the numerical coefficients come from eq. (5.2). In two dimensions there is no EinsteinHilbert action if we sum over surfaces with a fixed topology so that only the total area $N_{2}$ survives in the action. One does not need to add any higher derivative couplings to the action, since these should correspond to irrelevant operators in the infrared, with vanishing coefficients at the fixed point of the infrared renormalization group. Indeed, in the continuum 
theory, the coefficients of possible $R^{2}$ and Weyl-squared terms in the action vanish at the conformal fixed point [25]. The trace anomaly induced action (4.53) should not be added to the bare lattice action (5.7) either. It is nonlocal in the full metric and should be generated dynamically by the quantum fluctuations of the simplicial geometries, in analogy with the situation in the two dimensional case.

Now the partition function or functional integral over four-geometries is defined in the dynamical triangulation approach as

$$
Z_{D T}\left(k_{2}, k_{4}\right) \equiv \sum_{\mathcal{T}} e^{-S(\mathcal{T})}=\sum_{N_{4}} Z\left(k_{2} ; N_{4}\right) e^{-k_{4} N_{4}} \sim \sum_{N_{4}} e^{-\left[k_{4}-k_{4}^{c}\left(k_{2}\right)\right] N_{4}}
$$

where the last step follows if the number of triangulations which can be made from a given number $N_{4}$ of 4-simplices of fixed topology is exponentially bounded with respect to $N_{4}$ [6.76]. This is an important condition upon which the entire viability of the dynamical triangulation approach depends. In four dimensions there is as yet no rigorous proof of this exponential bound, and further work is necessary. If the exponential bound on the number of triangulations with fixed topology and fixed $N_{4}$ holds, then the partition function (5.9) must exist in a region of the coupling constant plane $k_{4}>k_{4}^{c}\left(k_{2}\right)$. By approaching the boundary of this region from above one can hope to arrive at a continuum limit in which physical correlation lengths go to infinity when expressed in lattice units. In this precise sense, one is searching for a "critical" curve in the $\left(k_{2}, k_{4}\right)$ plane corresponding to the existence of the a non-trivial infinite volume limit of the lattice theory defined by (5.7) and (5.8). Only on this curve, and as we shall see in a moment, only at one point on this curve can one hope to find a second order phase transition where correlation lengths go to infinity in units of the lattice spacing and the continuum limit of the theory may be defined.

We are interested in the continuum infrared fixed point of the lattice theory, which can be obtained, in principle, by block averaging over larger and larger sublattices, thereby determining the mapping from the bare lattice action with parameters $\left(k_{2}, k_{4}\right)$ to the renormalized parameters of the effective action at larger and larger distance scales. The fixed point of this mapping, if it exists, is the infrared fixed point of the continuum theory. In practice, 
in the dynamical triangulation approach one does not need to perform the block averaging procedure explicitly, since by taking more and more four simplices $\left(i . \quad\right.$ e. $\left.N_{4} \rightarrow \infty\right)$, one is effectively averaging over larger and larger sublattices in any case. Provided the exponential bound applies we can write down the finite volume scaling relation in the dynamical triangulation approach by simply translating the continuum relation (4.84) to the lattice, and obtain

$$
Z\left(k_{2} ; N_{4}\right) \simeq \tilde{Z}\left(\tilde{k}_{2}\right) N_{4}^{-\frac{Q^{2}}{2 \beta}-1} e^{k_{4}^{c}\left(k_{2}\right) N_{4}}
$$

where

$$
\tilde{k}_{2}=k_{2} N_{4}^{\frac{\alpha}{2 \beta}}
$$

in the case of fixed $S^{4}$ topology. The import of this relation for the lattice simulations is that if $k_{2}$ is scaled to zero as $N_{4} \rightarrow \infty$, with $\tilde{k}_{2}$ fixed, then the fixed volume partition function $Z\left(k_{2} ; N_{4}\right)$ should scale to zero with a definite power of $N_{4}$ that depends only on $Q^{2}$. If $Q^{2}$ were calculated reliably for gravitons in perturbation theory there are no free parameters in this prediction. Therefore, a clear test of this scaling relation is that the power of $N_{4}$ must be independent of the parameter $\tilde{k}_{2}$, if our hypothesis of infrared conformal dominance is correct.

We are proposing therefore that the procedure for finding the infrared fixed point of quantum gravity in the continuum limit from the dynamical triangulation starting point is to first locate the critical curve in the $\left(k_{2}, k_{4}\right)$ plane. Then one should attempt to run simulations with even larger volumes, moving along the critical curve towards the origin by rescaling $k_{2} \rightarrow 0$ as $N_{4} \rightarrow \infty$ in accordance with (5.11), keeping $\tilde{k}_{2}$ fixed. In this limit one can then test the validity of the scaling behavior for pure quantum gravity at its infrared fixed point. Preliminary results of the simulations with $N_{4} \sim 10^{4}$, seem to indicate a critical curve $k_{4}^{c}\left(k_{2}\right)$ which is approximately linear with a slope slightly more than 2 [31]. This is encouraging since the approximate slope of the critical curve should be $5 \theta / \pi=2.097847$ near the infrared critical point at the origin, according to $(5.8)$, 


$$
k_{4}^{c}\left(k_{2}\right)=\frac{5 \theta}{\pi} k_{2}+\frac{\sqrt{5}}{18 \pi}\left(\kappa^{2} \lambda\right)\left(k_{2}\right)^{2}
$$

but more data on bigger simplices are needed to confirm this.

In addition to the scaling of the finite volume partition function, one may consider a variety of physical observables as well. The simplest observable with nice scaling behavior is the average curvature $\langle R\rangle$ defined by $[6]$ :

$$
\langle R\rangle \equiv\left\langle\frac{\int d^{4} x \sqrt{g} R}{\int d^{4} x \sqrt{g}}\right\rangle=\frac{2 \pi V_{2}}{V_{4}}\left[\frac{1}{N_{4}} \frac{\partial}{\partial k_{2}} \ln Z\left(k_{2} ; N_{4}\right)-\frac{5 \theta}{\pi}\right],
$$

where the last equality holds for fixed volume and we used the relations (5.3), (5.5) and (5.7). Now inserting the scaling behavior (5.10) and the expression (5.12) one obtains

$$
\langle R\rangle \propto k_{2} \propto N_{4}^{-\alpha / 2 \beta} \rightarrow 0
$$

where the proportionality factor depends only on $Q^{2}$ and the rescaled $\tilde{k}_{2}$. This shows that the average curvature scales to zero, which is very different behaviour from the classical Einstein theory where $\langle R\rangle$ is simply proportional to the cosmological constant. This is a different way of seeing that the effective cosmological term vanishes in the infinite volume scaling limit. One question that has arisen in applying (5.14) to the available numerical data with finite volume lattices is whether subtractive normalizations to (5.14) are necessary. This is an important point which requires further elucidation, since without it we do not yet know how to compare the numerical data directly to the theoretical prediction and interpret the results.

Similar considerations apply for the curvature-curvature correlator

$$
\begin{aligned}
\int d^{4} x \sqrt{g}\langle R(x) R(0)\rangle & \sim \frac{1}{N_{4}}\left\langle\left(N_{2}-\frac{5}{\pi} \theta N_{4}\right)^{2}\right\rangle \\
& =\frac{1}{N_{4} Z}\left(\frac{\partial}{\partial k_{2}}-\frac{5}{\pi} \theta N_{4}\right)^{2} Z\left(k_{2} ; N_{4}\right) .
\end{aligned}
$$

If we substitute (5.12), (5.10) and (5.11) into this last expression, a short calculation shows this correlation function also goes to a finite constant, independent of $N_{4}$ in the large volume limit. This means that the correlator $\langle R(x) R(0)\rangle$ must fall off faster than $|x|^{-4}$ for large 
$|x|$, in order for the integral over $x$ to converge. Contrast this convergent behavior of (5.15) in the conformal invariant phase of $4 \mathrm{D}$ gravity to the behavior of the same quantity in the classical theory. In that case the trace of the classical Einstein equations, $R=4 \Lambda$ rigidly fixes the scalar curvature in terms of the cosmological constant. Hence (5.15) must diverge linearly with the volume $N_{4}$ in the classical theory, if the cosmological term is non-vanishing.

We conclude that if the quantum fluctuations of the conformal factor described in this article really dominate in the far infrared, then any non-zero cosmological term in the continuum theory is screened completely at the largest distance scales by these fluctuations, or in other words, the effective cosmological constant in the scale invariant vacuum of $4 \mathrm{D}$ quantum gravity must vanish.

\section{CONCLUSIONS AND OPEN PROBLEMS}

In this article the invariant functional integral for coordinate invariant systems has been constructed and exploited to yield a variety of results, some previously known and some new. The measure in the coset functional space of metrics modulo spacetime diffeomorphisms has been determined by a geometric construction, and agrees with other methods. The invariant path integral framework shows clearly the problems with naive semi-classical expansions around spaces with normalizable Killing vector fields such as de Sitter space, where the measure vanishes, and infrared instabilities appear in the conformal factor of the metric. The effective theory of the conformal factor in four dimensions constructed also by means of the invariant integral for gravity provides a novel insight into the importance of quantum effects in gravitation at large distance scales, whose physical consequences are still under investigation. The continuum definition of the sum over geometries provides a useful framework for extracting information about quantum gravity at its large volume infrared fixed point, independently of the underlying theory at the Planck scale. This is seen explicitly in the finite volume scaling relations and comparison to the dynamical triangulation

approach. The application of methods from statistical mechanics and critical phenomena to 
qunatum gravity is made possible by the invariant functional integral formulation.

Many issues remain to be explored further. On a formal level, it is important to place the construction of the measure on coset spaces $\mathcal{M} \mathcal{G}$ on a more rigourous mathematical foundation. The connection of this construction to canonical phase space methods has yet to be fully elucidated. Global issues of topology and coordinate charts on this very large space have been scarcely touched in this article or the literature in general. It is important to know if there are global obstructions to the construction and what the consequences of such obstructions to physics might be. The Yamabe uniformization problem and classification of geometries in four dimensions clearly require much more work as well. Any extension of understanding or exact results for functional integrals other than Gaussian would definitely be welcome. Investigation of the simplicial definition of the functional integral is also needed. Is there indeed an exponential bound to the partition function at fixed topology and fixed volume? What is the measure in this space of simplices and does it approximate the continuum measure correctly at large volumes? How should the finite volume numerical results be compared to the theoretical predictions which can hold strictly only at the infrared fixed point in the infinite volume limit? Analytic work as well as more efficient algorithms for numerical simulations on bigger lattices are both necessary in the dynamical triangulation framework.

Of the physics issues raised, a calculation of the graviton contribution to the infrared anomaly and finite volume scaling is clearly paramount. A clean test of the scaling relations by the numerical simulations would be the next most important development, possibly providing a complete solution of the long-standing cosmological constant problem [32]. Such a dynamical mechanism based on infrared conformal invariance, internal to the structure of quantum gravity itself with no additional assumptions is very appealing and would herald a new era in cosmology and large scale structure formation. The invariant functional integral over geometries appears to provide us with the most powerful tool to date for prying open the door at least a bit to a better understanding of quantum gravity. 


\section{REFERENCES}

[1] K. G. Wilson and J. Kogut, Phys. Rep. C12 (1974) 75;

K. G. Wilson, Rev. Mod. Phys. 47 (1975) 773.

[2] A. M. Polyakov, Phys. Lett. B103 (1981) 207, 211; Gauge Fields and Strings, (Harwood Academic, Chur, 1987).

[3] P. O. Mazur and E. Mottola, Nucl. Phys. B341 (1990) 187.

[4] S. K. Blau, Z. Bern, and E. Mottola, Phys. Rev. D43 (1991) 1212;

P. O. Mazur, Phys. Lett. B262 (1991) 405.

[5] J. Ambjørn, B. Durhuus, J. Frohlich and P. Orland, Nucl. Phys. B270 (1986) 457;

A. Billoire and F. David, Nucl. Phys. B275 (1986) 617;

D. V. Boulatov, V. A. Kazakov, I. K. Kostov and A. A. Migdal, Nucl. Phys. B275 (1986) 641.

[6] J. Ambjørn and J. Jurkiewicz, Phys. Lett. B278 (1992) 42;

J. Ambjørn, Act. Phys. Pol. B23(1992) 991;

J. Ambjørn, J. Jurkiewicz, and C. Kristjansen, Nucl. Phys. B393 (1993) 601.

[7] M. E. Agishtein and A. A. Migdal, Mod. Phys. Lett. A7 (1992) 1039; Nucl. Phys. B385 (1992) 395 .

[8] I. Antoniadis, P. O. Mazur and E. Mottola, Phys. Lett. B323 (1994) 284.

[9] I. M. Gel'fand and A. M. Yaglom, Jour. Math. Phys. 1 (1960) 1;

R. P. Feynman and A. R. Hibbs, Quantum Mechanics and Path Integrals, (McGraw-Hill, New York, 1965).

[10] E. S. Fradkin and G. A. Vilkovisky, Phys. Lett. B55 (1975) 2;

I. A. Batalin and G. A. Vilkovisky, Phys. Lett. B69 (1977) 309; Nucl. Phys. B234 (1984) 106 . 
[11] O. Babelon and C.-M. Vaillet, Phys. Lett. B85 (1979) 246; Comm. Math. Phys. (1981) 515.

[12] L. C. L. Botelho, Phys. Rev. D38 (1988) 2464;

P. Ellicott, G. Kunstatter and D.J. Toms, Mod. Phys. Lett. A4 (1989) 2397.

[13] V. N. Gribov, Nucl. Phys. B139 (1978) 1.

[14] K. Fujikawa, Phys. Rev. Lett. 42 (1979) 1195; 44 (1980) 1733;

Nucl. Phys. B226 (1983) 437;

K. Fujikawa and O. Yasuda, Nucl. Phys. B245 (1984) 436.

[15] H. Yamabe, Osaka Math. J. 12 (1960) 21;

T. Aubin, Jour. Diff. Geom. 4 (1970) 383; Jour. Math. Pure and Appl. 55 (1976) 269;

Nonlinear Analysis on Manifolds: Monge-Ampère Equations (Springer, New York 1982);

M. S. Berger, Jour. Diff. Geom. 5 (1971) 325;

R. Schoen, Jour. Diff. Geom. 20 (1984) 479;

[16] B. S. DeWitt, General Relativity: An Einstein Centenary Survey, S. W. Hawking and W. Israel editors, (Cambridge Univ. Press, Cambridge, 1979); Relativity, Groups and Topology II, B. S. DeWitt and R. Stora editors, (North-Holland, Amsterdam, 1984).

[17] S. Deser, M. J. Duff, and C. J. Isham, Nucl. Phys. B111 (1976) 45;

N. D. Birrell and P. C. W. Davies, Quantum Fields in Curved Space, (Cambridge Univ. Press, Cambridge, 1982).

[18] E. D'Hoker and D. H. Phong, Rev. Mod. Phys. 60 No. 4 (1988) 917.

[19] S. W. Hawking, Phys. Rev. D18 (1978) 1747;

Recent Developments in Gravitation, M. Levy and S. Deser eds. (Plenum, New York, 1979);

General Relativity: An Einstein Centennial Survey, S. W. Hawking and W. Israel eds. (Cambridge University Press, Cambridge, 1979). 
G. W. Gibbons, S. W. Hawking and M. J. Perry, Nucl. Phys. B138 (1978) 141.

[20] D. V. Vassilevich, Intl. J. Mod. Phys. A8 (1993) 1637.

[21] E. Mottola, Phys. Rev. D31 (1986) 754; Phys. Rev D33 1616; ibid. 2136;

I. Antoniadis and E. Mottola, (1991) Jour. Math. Phys. 32, 1037.

[22] V. Moncrief, Jour. Math. Phys. 16 (1975) 493; ibid. 1556; 17 (1976) 1893.

[23] M. J. Duff, Nucl. Phys. B125 (1977) 334.

[24] R. J. Riegert, Phys. Lett. B134 (1984) 56;

E. S. Fradkin and A. A. Tseytlin, Phys. Lett. B134 (1984) 187;

S. Odintsov and I. Shapiro, Class. Quant. Grav. 3 (1991).

[25] I. Antoniadis and E. Mottola, Phys. Rev. D45 (1992) 2013.

I. Antoniadis, P. O. Mazur, and E. Mottola, Nucl. Phys. B 388 (1992) 627.

[26] F. David, Mod. Phys. Lett. A3 (1988) 1651;

J. Distler and H. Kawai, Nucl. Phys. B321 (1989) 509.

[27] V. G. Knizhnik, A. M. Polyakov, and A. B. Zamolodchikov, Mod. Phys. Lett. A3 (1988) 819.

[28] C. Schmidhuber, Nucl. Phys. B390 (1993) 188.

[29] N. H. Christ, R. Friedberg, and T. D. Lee, Nucl. Phys. B202 (1982) 89.

[30] J. Ambjørn and J. Jurkiewicz, Neils Bohr Inst. preprint, NBI-HE-94-29, (May 1994) [e-Print Archive: hep-lat@ftp.scri.fsu.edu - 9405010]

B. V. De Bakker and J. Smit, Phys. Lett. B334 (1994) 304.

[31] J. Ambjørn, private communication.

[32] S. Weinberg, Rev. Mod. Phys. 61 (1989) 1. 